\title{
Peridynamic Thermal Diffusion
}

Selda Oterkus and Erdogan Madenci*

Department of Aerospace and Mechanical Engineering, The University of Arizona, Tucson, AZ 85721,USA

Abigail Agwai

Intel Corporation, Chandler, AZ 85226,USA

\begin{abstract}
This study presents the derivation of ordinary state-based peridynamic heat conduction equation based on the Lagrangian formalism. The peridynamic heat conduction parameters are related to those of the classical theory. An explicit time stepping scheme is adopted for numerical solution of various benchmark problems with known solutions. It paves the way for applying the peridynamic theory to other physical fields such as neutronic diffusion and electrical potential distribution.
\end{abstract}

\section{Introduction}

Nonlocal theories have been used to some extent to describe heat conduction on a continuum level. In heat conduction, the thermal energy is transported through phonon, lattice vibration, and electrons. Usually, electrons are the vehicles through which thermal energy is transported in metals while phonons are the heat carrier in insulator and semiconductors. This process of thermal energy transfer is inherently nonlocal because the carriers arrive at one point having brought thermal energy from another. Nevertheless, macroscale heat transfer models that adopt a local formulation, typically employing the Fourier's law as the local constitutive relation, have been used successfully to represent continuum heat conduction.

The mean free path of the heat carriers is the average distance a carrier travels before its excess energy is lost. As the heat carriers' mean free path becomes comparable to the characteristic lengths; the nonlocality needs to be taken into account in the continuum model. Nonlocality often becomes important at low temperatures, as exhibited in cryogenics systems, since the heat carriers have a longer mean free path at lower temperatures. It has been found that nonlocality should also be accounted for in problems in which the temperature gradients are steep. This is because the penetration depth, the length characterizing the temperature gradient, becomes short, even becoming the same order of magnitude as the mean free path of the carrier. In such instances, it is necessary to consider the nonlocality of the heat transport in a continuum model. Recently, with the miniaturization of devices, the small geometric length scales have also necessitated the inclusion of nonlocal effects in microscale and nanoscale models [1].

Keywords: Thermal, Conduction, Diffusion, Peridynamics, Nonlocal

*Corresponding author. Phone: 00+1+520-621-6113. Fax: 00+1+520-621-8191.

E-mail address: madenci@email.arizona.edu. 
Several nonlocal heat conduction theories have been proposed in the last few decades. In the early 1980s, Luciani et al. [2] developed a nonlocal theory to better represent electron heat transport down a steep temperature gradient by introducing a nonlocal expression for the heat flux. The nonlocal model was in better agreement with probabilistic simulations (Fokker-Planck simulations) than the local models. Later, Mahan and Claro [3] proposed a nonlocal relation between the heat current, determined from Boltzmann's equation, and the temperature gradient. In the 1990s, Sobolev [4] introduced a model in which both space and time nonlocality are taken into account in the strong form, i.e. integral form, of the energy balance, Gibbs and entropy balance equations. Lebon and Grmela [5], proposed a weakly nonlocal model (weakly nonlocal are typically based on gradient formulation). The model was based on nonequilibrium thermodynamics, for which an extra variable is added to the basic state variables to account for nonlocality. Subsequently, they extended their model to include nonlinearity [6]. More recently, the development of nonlocal heat conduction equations has been motivated by the miniaturization of devices. A number of researchers have put forth nonlocal models with the objective of capturing heat transport in microscale and nanoscale devices. One example of this is the ballistic-diffusive heat equation by Chen [7], which was derived from the Boltzmann's equation, and it accounts for nonlocality in heat transport. Another example is by Alvarez and Jou [8]. They developed their model by including nonlocal (and memory/lag) effects in irreversible thermodynamics. Tzou and Guo [9] constructed their model by incorporating a nonlocal (and lag) term into the Fourier law.

An area of interest is determining the temperature field in the presence of emerging discontinuities. One class of problems that contains a discontinuity is the heat transfer process which involves phase change such as solidification and melting [10]. This process is commonly referred to as Stefan problem, and there are a number of technologically important problems that involve heat transfer with phase change. Examples of these include ablation of space vehicles during reentry and casting of metals. Another heat conduction problem with an emerging discontinuity is the rewetting problem from the nuclear industry. Rewetting in a nuclear reactor is employed to restore temperatures to a safe range following accidental dry out or loss of coolant. Emergency cooling is introduced to the system via an upward moving water front or by spraying from the top of the reactor $[11,12]$. A moving discontinuity occurs in the heat generating solid at the quench front due to the sudden change in heat transfer condition at the solid surface.

Peridynamics is a nonlocal continuum theory which allows governing field equations to be applicable at discontinuities. This applicability at discontinuities is achieved by replacing the spatial derivatives, which lose meaning at discontinuities, with integrals that are valid regardless of the existence of a discontinuity. A peridynamic heat conduction model allows problems with discontinuities are readily solvable as no spatial derivatives appear in the formulation, making the equation applicable everywhere in the body.

The peridynamic theory was initially developed as a reformulation of the equation of motion in solid mechanics that was better suited for modeling bodies with discontinuities, such as cracks [13]. The theory was formulated in what is now referred as the bond- 
based peridynamic theory, in which a body assumed to be comprised of a network of independent pairwise interactions. However, the independence of the pairwise interactions in solid mechanics leads to certain material limitation. As a result, Silling et al. [14] and Silling and Lehoucq [15] developed a generalized approach to peridynamics in which interactions are not independent, and referred to as state-based peridynamics. Peridynamic states were introduced as the mathematical objects that convey the information associated with a body. Within the realm of solid mechanics, the peridynamic theory has been successfully employed to model fracture nucleation and propagation [16].

A peridynamic approach to heat conduction is advantageous as it not only accounts for nonlocality but it also allows for the determination of the temperature field in spite of discontinuities. The peridynamic heat conduction model is a continuum model; it is not a discrete model. As such the phonon and electron motion is not explicitly modeled. Initial successful attempts have recently been made to develop heat conduction equations in the peridynamic framework. Gerstle et al. [17] developed a peridynamic model for electromigration that accounts for heat conduction in a one dimensional body. Additionally, Bobaru and Duangpanya [18] proposed a one dimensional peridynamic heat conduction equation. Recently, Bobaru and Duangpanya also solved the 2-D heat conduction problem with discontinuities [19]. Both studies adopted the bond-based peridynamic approach.

As part of this study, the heat conduction equation is formulated within the framework of generalized state-based peridynamics. To begin with the peridynamic states are reviewed. The derivation of the generalized peridynamic heat equation is demonstrated using the Lagrangian formalism and the peridynamic variables are explained. Subsequently, simplifications are made to develop the bond-based peridynamic approach for heat conduction from the generalized state-based. The thermal response function and an approach for determining the microconductivity are also presented. A numerical procedure is described for solving the peridynamic heat conduction equations along with the discretization and time stepping schemes as well as numerical stability criterion. Various problems are simulated based upon the present peridynamic heat transfer model, and comparisons against classical solutions are presented in order to establish its validity.

\section{State-Based Peridynamic Thermal Diffusion Equation}

Within the peridynamic framework, the interaction between material points is nonlocal. For thermal diffusion, the nonlocal interaction between material points is due to the exchange of heat energy. Therefore, a material point will exchange heat with points within its neighborhood defined by the horizon.

In the Lagrangian formalism, the governing heat conduction equation corresponds to the Euler-Lagrange equation. The Euler-Lagrange equation based on the Lagrangian , $L$ is given in the following form [20] 
$\frac{d}{d t}\left(\frac{\partial L}{\partial \dot{\Theta}}\right)-\frac{\partial L}{\partial \Theta}=0$

with

$L=\int_{V} \mathfrak{L} d V$

in which $\Theta$ is the temperature, $\mathfrak{L}$ is the lagrangian density. The Lagrangian density of a peridynamic material point can be defined as

$\mathfrak{L}=Z+\rho \widehat{s} \Theta$

where $Z$ is thermal potential and it is a function of all the temperatures of the points that $\mathbf{x}$ interacts with, $\rho$ is the density and $\hat{s}$ is the heat source per unit mass, which includes the rate of heat generation per unit volume and the internal energy storage. There is a thermal potential associated with each material point, and the term, $Z_{(i)}$ represents the thermal potential of material point, $\mathbf{x}_{(i)}$. The microthermal potential, $z_{(i)(j)}$ is the thermal potential due to the interaction (exchange of heat energy) between material point, $\mathbf{x}_{(i)}$ and $\mathbf{x}_{(j)}$. The microthermal potential is related to heat energy exchange, which depends on the temperature difference between the material points. Therefore, the microthermal potential is dependent on the temperature difference between pairs of material points. More specifically, the microthermal potential, $z_{(i)(j)}$ depends on the temperature difference between point, $i$ and all other material points that interact with point, $\mathbf{x}_{(i)}$. Note that the microthermal potential $z_{(j)(i)} \neq z_{(i)(j)}$, as $z_{(j)(i)}$ depends on the state of material points that interact with material point, $\mathbf{x}_{(j)}$. The microthermal potential is denoted as follows,

$$
z_{(i)(j)}=z_{(i)(j)}\left(\Theta_{\left(1^{i}\right)}-\Theta_{i}, \Theta_{\left(2^{i}\right)}-\Theta_{i}, \cdots\right) \quad z_{(j)(i)}=z_{(j)(i)}\left(\Theta_{\left(1^{j}\right)}-\Theta_{j}, \Theta_{\left(2^{j}\right)}-\Theta_{j}, \cdots\right)
$$

where $\Theta_{(i)}$ is the temperature at point, $\mathbf{x}_{(i)}$ and $\Theta_{\left(1^{i}\right)}$ is the temperature of the first material point that interacts with point, $\mathbf{x}_{(i)}$, and similarly, $\Theta_{(j)}$ is the temperature at point, $\mathbf{x}_{(j)}$ while $\Theta_{\left(1^{j}\right)}$ is the temperature of the first material point that interacts with point, $\mathbf{x}_{(j)}$ as shown in Fig. 1. 


\begin{tabular}{|c|c|c|c|c|c|c|c|c|c|c|c|}
\hline & & & & & & 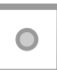 & & & 0 & 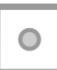 & \\
\hline & 0 & $H$ & 0 & & $\rightarrow$ & צ. & 0 & & 0 & o & \\
\hline & $c$ & & & & 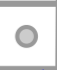 & 0 & $\therefore$ & ? & 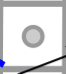 & $H_{\mathbf{x}_{(j)}}$ & \\
\hline & $c$ & $c$ & & & $D$ & & 0 & $p$ & $a$ & 0 & \\
\hline & O & $\Phi$ & & d & $\boldsymbol{\circ i}$ & 0 & 0 & 1 & 0 & D & \\
\hline \multicolumn{4}{|c|}{ in $N+\sqrt{i}$} & & 0 & 0 & $j a$ & P & 0 & p & 0 \\
\hline & t & 0 & $p$ & a & 0 & ○ & 8 & \multicolumn{4}{|c|}{$k-1 / K^{\prime} k+1$} \\
\hline & 0 & 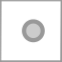 & 0 & 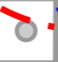 & $\vee$ & 0 & ㅇ & 0 & 9 & 0 & 0 \\
\hline & 이 & 0 & 0 & 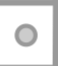 & 0 & 7 & $\theta$ & $\sigma$ & 0 & 0 & 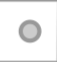 \\
\hline & & & & & 0 & 0 & $\circ$ & 0 & 0 & 0 & \\
\hline
\end{tabular}

Figure 1. Peridynamic material points and their interaction with each other

The thermal potential of point, $\mathbf{x}_{(i)}, Z_{(i)}$ is defined as

$$
\begin{aligned}
Z_{(i)}= & \frac{1}{2} \sum_{j=1}^{\infty} \frac{1}{2}\left(z_{(i)(j)}\left(\Theta_{\left(1^{i}\right)}-\Theta_{(i)}, \Theta_{\left(2^{i}\right)}-\Theta_{(i)}, \cdots\right)\right. \\
& \left.+z_{(j)(i)}\left(\Theta_{\left(1^{j}\right)}-\Theta_{(j)}, \Theta_{\left(2^{j}\right)}-\Theta_{(j)}, \cdots\right)\right) V_{(j)}
\end{aligned}
$$

where $V_{(j)}$ is the infinitesimal volume associated with material point, $\mathbf{x}_{(j)}$. Basically, this equation indicates that the thermal potential at a point is the summation over all the microthermal potential associated with that point. The Euler-Lagrange equation, Eq. (1a), for material point, $\mathbf{x}_{(k)}$ becomes

$\frac{d}{d t}\left(\frac{\partial L}{\partial \dot{\Theta}_{(k)}}\right)-\frac{\partial L}{\partial \Theta_{(k)}}=0$.

in which

$$
L=\sum_{i=1}^{\infty} \mathfrak{L}_{(i)} V_{(i)} .
$$

with

$$
\mathfrak{L}_{(i)}=Z_{(i)}+\rho \widehat{s}_{(i)} \Theta_{(i)} .
$$


The microthermal potential and therefore thermal potential are both functions of temperature. Consequently, invoking Eq. (2) and Eq. (4) into Eq. (5a) results in the following equation

$$
\begin{aligned}
& \left(\sum_{j=1}^{\infty} \frac{1}{2}\left(\sum_{i=1}^{\infty} \frac{\partial z_{(k) i)}}{\partial\left(\Theta_{(j)}-\Theta_{(k)}\right)} V_{(i)}\right) \frac{\partial\left(\Theta_{(j)}-\Theta_{(k)}\right)}{\partial \Theta_{(k)}}\right. \\
& \left.\quad+\sum_{j=1}^{\infty} \frac{1}{2}\left(\sum_{i=1}^{\infty} \frac{\partial z_{(i)(k)}}{\partial\left(\Theta_{(k)}-\Theta_{(j)}\right)} V_{(i)}\right) \frac{\partial\left(\Theta_{(k)}-\Theta_{(j)}\right)}{\partial \Theta_{(k)}}\right) V_{(k)}+\rho \widehat{s}_{(k)} V_{(k)}=0
\end{aligned}
$$

or

$$
-\sum_{j=1}^{\infty} \frac{1}{2}\left(\sum_{i=1}^{\infty} \frac{\partial z_{(k)(i)}}{\partial\left(\Theta_{(j)}-\Theta_{(k)}\right)} V_{(i)}\right)+\sum_{j=1}^{\infty}\left(\sum_{i=1}^{\infty} \frac{\partial z_{(i)(k)}}{\partial\left(\Theta_{(k)}-\Theta_{(j)}\right)} V_{(i)}\right)+\rho \widehat{s}_{(k)}=0
$$

in which the terms $\sum_{i=1}^{\infty} V_{(i)} \partial z_{(k)(i)} / \partial\left(\Theta_{(j)}-\Theta_{(k)}\right)$ and $\sum_{i=1}^{\infty} V_{(i)} \partial z_{(i)(k)} / \partial\left(\Theta_{(k)}-\Theta_{(j)}\right)$ can be thought of as the heat flow density from material point, $\mathbf{x}_{(j)}$ to material point, $\mathbf{x}_{(k)}$ and the heat flow density from material point $\mathbf{x}_{(k)}$ to $\mathbf{x}_{(j)}$, respectively. Based on this interpretation, $\mathcal{H}_{(k)(j)}$ and $\mathcal{H}_{(j)(k)}$ are introduced, and defined as

$$
\mathcal{H}_{(k)(j)}=\frac{1}{2} \frac{1}{V_{(j)}}\left(\sum_{i=1}^{\infty} \frac{\partial z_{(k)(i)}}{\partial\left(\Theta_{(j)}-\Theta_{(k)}\right)} V_{(i)}\right),
$$

and

$$
\mathcal{H}_{(j)(k)}=\frac{1}{2} \frac{1}{V_{(j)}}\left(\sum_{i=1}^{\infty} \frac{\partial z_{(i)(k)}}{\partial\left(\Theta_{(k)}-\Theta_{(j)}\right)} V_{(i)}\right),
$$

Using these definitions allows Eq. (6b) to be rewritten as follows

$\sum_{j=1}\left(-\mathcal{H}_{(k)(j)}+\mathcal{H}_{(j)(k)}\right) V_{(j)}+\rho \widehat{s}_{(k)}=0$

As described by Silling et al [14] and Silling and Lehoucq [15], a PD state can be thought of as an infinite dimensional array that contains certain information about all the interactions associated with a particular material point. All of the heat flow density associated with each interaction assembled in an infinite-dimensional array is referred to as the heat flow scalar state, $\underline{h}(\mathbf{x}, t)$ where $t$ is the time. The assembled heat flow state for material points $\mathbf{x}_{(k)}$ to $\mathbf{x}_{(j)}$ may be represented as 
$\underline{h}\left(\mathbf{x}_{(k)}, t\right)=\left\{\begin{array}{c}\vdots \\ \mathcal{H}_{(k)(j)} \\ \vdots\end{array}\right\} \quad$ and $\quad \underline{h}\left(\mathbf{x}_{(j)}, t\right)=\left\{\begin{array}{c}\vdots \\ \mathcal{H}_{(j)(k)} \\ \vdots\end{array}\right\}$

The heat flow state associates each pair of interacting material points with a heat flow density, and enables the expressions for heat flow densities $\mathcal{H}_{(k)(j)}$ and $\mathcal{H}_{(j)(k)}$ as

$$
\mathcal{H}_{(k)(j)}=\underline{h}\left(\mathbf{x}_{(k)}, t\right)\left\langle\mathbf{x}_{(j)}-\mathbf{x}_{(k)}\right\rangle \quad \text { and } \quad \mathcal{H}_{(j)(k)}=\underline{h}\left(\mathbf{x}_{(j)}, t\right)\left\langle\mathbf{x}_{(k)}-\mathbf{x}_{(j)}\right\rangle,
$$

where the angled brackets include the interacting material points. The microthermal potentials may also be assembled in a state, which is called the microthermal potential scalar state, $\underline{z}(\mathbf{x}, t)$, permitting the following representation

$$
z_{(k)(j)}=\underline{z}\left(\mathbf{x}_{(k)}, t\right)\left\langle\mathbf{x}_{(j)}-\mathbf{x}_{(k)}\right\rangle \text { and } z_{(j)(k)}=\underline{z}\left(\mathbf{x}_{(j)}, t\right)\left\langle\mathbf{x}_{(k)}-\mathbf{x}_{(j)}\right\rangle \cdot
$$

Utilizing the concept of PD states, Eq. (7) may be rewritten as

$$
\underline{h}=\frac{1}{2} \frac{\partial \underline{z}}{\partial\left(\Theta_{(j)}-\Theta_{(k)}\right)} .
$$

Applying the state notation, Eq. (8) can be also rewritten as

$$
\sum_{j=1}^{\infty}\left(\underline{h}\left(\mathbf{x}_{(k)}, t\right)\left\langle\mathbf{x}_{(j)}-\mathbf{x}_{(k)}\right\rangle-\underline{h}\left(\mathbf{x}_{(j)}, t\right)\left\langle\mathbf{x}_{(k)}-\mathbf{x}_{(j)}\right\rangle\right) V_{(j)}-\rho \widehat{s}_{(k)}=0
$$

Because the volume of each material point $V_{(j)}$ is infinitesimally small, for the limiting case of $V_{(j)} \rightarrow 0$, the summation can be replaced with integration over the material points within the horizon as

$$
\sum_{j=1}(\cdot) V_{(j)} \rightarrow \int_{H}(\cdot) d V_{\mathbf{x}^{\prime}}
$$

It permits Eq. (13) to be recast as

$$
\int_{H}\left(\underline{h}(\mathbf{x}, t)\left\langle\mathbf{x}^{\prime}-\mathbf{x}\right\rangle-\underline{h}\left(\mathbf{x}^{\prime}, t\right)\left\langle\mathbf{x}-\mathbf{x}^{\prime}\right\rangle\right) d V_{\mathbf{x}^{\prime}}-\rho \widehat{s}=0,
$$

where $\underline{h}(\mathbf{x}, t)\left\langle\mathbf{x}^{\prime}-\mathbf{x}\right\rangle=0$ for $\mathbf{x}^{\prime} \notin H$, and domain of integration, $H$ is defined by the horizon of the material point, $\mathbf{x}$ that interacts with other material points in its own family. 
For convenience, the following notation is adopted

$\underline{h}(\mathbf{x}, t)=\underline{h}, \quad \underline{h}\left(\mathbf{x}^{\prime}, t\right)=\underline{h}^{\prime}$.

Also, the temperature scalar state, $\underline{\tau}$ is defined as

$\underline{\tau}(\mathbf{x}, t)\left\langle\mathbf{x}^{\prime}-\mathbf{x}\right\rangle=\Theta\left(\mathbf{x}^{\prime}, t\right)-\Theta(\mathbf{x}, t)$.

The temperature state simply contains the temperature difference associated with each interaction of a particular material point. Since, the microthermal potential is dependent on the temperature difference of all the interactions associated with the material point, it may be written as a function of the temperature state,

$\underline{z}=\underline{z}(\underline{\tau})$.

Therefore, the heat flow state can also be written as a function of the temperature state,

$\underline{h}=\underline{h}(\underline{\tau})$.

As outlined by Bathe [21], the heat conduction equation should explicitly include the rate at which heat energy is stored when the heat flow changes over a short period of time. This rate of internal energy storage density, $\dot{\varepsilon}_{s}$, is a negative energy source and it is given by

$\dot{\varepsilon}_{s}=c_{v} \frac{\partial \Theta}{\partial t}$

for which $c_{v}$ is the specific heat capacity.

Therefore, the source term in Eq. (13) is then replaced by $\widehat{s}=\dot{\varepsilon}_{s}-s_{b}$, where $s_{b}$ is the heat source due to volumetric heat generation per unit mass. Invoking Eq. (20) into Eq. (13) leads to the transient form of the state-based peridynamic thermal diffusion equation

$\rho c_{v} \dot{\Theta}(\mathbf{x}, t)=\int_{H} \underline{h}(\mathbf{x}, t)\left\langle\mathbf{x}^{\prime}-\mathbf{x}\right\rangle-\underline{h}\left(\mathbf{x}^{\prime}, t\right)\left\langle\mathbf{x}-\mathbf{x}^{\prime}\right\rangle d V^{\prime}+h_{s}(\mathbf{x}, t)$,

in which $h_{s}(\mathbf{x}, t)=\rho s_{b}(\mathbf{x}, t)$ is the heat source due to volumetric heat generation. The resulting equation is an integro-differential equation in time and space. It contains differentiation with respect to time, and integration in spatial domain. It does not contain any spatial derivatives of temperature; thus, the PD thermal equation is valid everywhere whether or not discontinuities exist in the domain. Construction of its solution involves time and spatial integrations while being subject to conditions on the boundary of the domain , $\mathcal{R}$, and initial conditions. 


\section{Relationship between heat flux and peridynamic heat flow state}

The heat flow scalar state, $\underline{h}$ contains the heat flow densities associated with all the interactions. The heat flow density, $\underline{h}(\mathbf{x}, t)\left\langle\mathbf{x}^{\prime}-\mathbf{x}\right\rangle$, has units of heat flow rate (rate of heat energy change) per volume square. The integral in Eq. (21),

$$
\int_{H} \underline{h}(\mathbf{x}, t)\left\langle\mathbf{x}^{\prime}-\mathbf{x}\right\rangle-\underline{h}\left(\mathbf{x}^{\prime}, t\right)\left\langle\mathbf{x}-\mathbf{x}^{\prime}\right\rangle d V^{\prime}
$$

is similar to the divergence of heat flux, $\nabla \cdot \mathbf{q}$, and it has units of heat flow rate per volume. Therefore, the peridynamic heat flow state can be related to the heat flux, $\mathbf{q}$.

Multiplying the PD heat conduction equation, Eq. (21), by a temperature variation of $\Delta \Theta$, and integrating over the entire domain result in

$$
\int_{V} \rho c_{v} \dot{\Theta} \Delta \Theta d V=\int_{V} \int_{H}\left[\underline{h}(\mathbf{x}, t)\left\langle\mathbf{x}^{\prime}-\mathbf{x}\right\rangle-\underline{h}\left(\mathbf{x}^{\prime}, t\right)\left\langle\mathbf{x}-\mathbf{x}^{\prime}\right\rangle\right] \Delta \Theta d V^{\prime} d V+\int_{V} h_{s}(\mathbf{x}, t) \Delta \Theta d V .
$$

After moving the last term on the right-hand side of Eq. (23), the heat generation term, to the left-hand side, and changing the integration from $H$ to $V$ due to the fact that

$\underline{h}(\mathbf{x}, t)\left\langle\mathbf{x}^{\prime}-\mathbf{x}\right\rangle=\underline{h}\left(\mathbf{x}^{\prime}, t\right)\left\langle\mathbf{x}-\mathbf{x}^{\prime}\right\rangle=0$ for $\mathbf{x}^{\prime} \notin H$,

leads to the following form of the equation

$$
\int_{V}\left[\rho c_{v} \dot{\Theta}-h_{s}(\mathbf{x}, t)\right] \Delta \Theta d V=\int_{V} \int_{V}\left[\underline{h}(\mathbf{x}, t)\left\langle\mathbf{x}^{\prime}-\mathbf{x}\right\rangle-\underline{h}\left(\mathbf{x}^{\prime}, t\right)\left\langle\mathbf{x}-\mathbf{x}^{\prime}\right\rangle\right] \Delta \Theta d V^{\prime} d V .
$$

If the parameters $\mathbf{x}$ and $\mathbf{x}^{\prime}$ in the second integral on the right-hand side of Eq. (25) are exchanged, the second integral

$$
\int_{V} \int_{V} \underline{h}\left(\mathbf{x}^{\prime}, t\right)\left\langle\mathbf{x}-\mathbf{x}^{\prime}\right\rangle \Delta \Theta d V^{\prime} d V=\iint_{V} \int_{V} \underline{h}(\mathbf{x}, t)\left\langle\mathbf{x}^{\prime}-\mathbf{x}\right\rangle \Delta \Theta^{\prime} d V d V^{\prime} .
$$

Substituting from Eq. (26) into Eq. (25), leads to

$$
\int_{V}\left[\rho c_{v} \dot{\Theta}-h_{s}(\mathbf{x}, t)\right] \Delta \Theta d V=\int_{V} \int_{V} \underline{h}(\mathbf{x}, t)\left\langle\mathbf{x}^{\prime}-\mathbf{x}\right\rangle\left(\Delta \Theta-\Delta \Theta^{\prime}\right) d V^{\prime} d V
$$

Invoking the variation of the temperature scalar state, $\Delta \underline{\tau}$ from Eq. (17) into Eq. (27) results in

$$
\int_{V}\left[\rho c_{v} \dot{\Theta}-h_{s}(\mathbf{x}, t)\right] \Delta \Theta d V=\int_{V} \Delta Z d V
$$


where $\Delta Z$ corresponds to the variation of the PD thermal potential at $\mathbf{x}$ due to its interactions with all other material points:

$$
\Delta Z=-\int_{V}\left(\underline{h}(\mathbf{x}, t)\left\langle\mathbf{x}^{\prime}-\mathbf{x}\right\rangle\right)\left(\Delta \underline{\tau}\left\langle\mathbf{x}^{\prime}-\mathbf{x}\right\rangle\right) d V^{\prime}
$$

Considering only the material points within the horizon, Eq. (29) can be rewritten as

$$
\Delta Z=-\int_{H}\left(\underline{h}(\mathbf{x}, t)\left\langle\mathbf{x}^{\prime}-\mathbf{x}\right\rangle\right)\left(\Delta \underline{\tau}\left\langle\mathbf{x}^{\prime}-\mathbf{x}\right\rangle\right) d V^{\prime}
$$

Based on the classical formulation, the corresponding variation of thermal potential can be written as

$$
\Delta \hat{Z}(\overline{\mathbf{G}})=\frac{1}{2}(\Delta \overline{\mathbf{G}} \cdot k \overline{\mathbf{G}}+\overline{\mathbf{G}} \cdot k \Delta \overline{\mathbf{G}})=k \overline{\mathbf{G}} \cdot \Delta \overline{\mathbf{G}} \cdot(31 \mathrm{a})
$$

with $\hat{Z}(\overline{\mathbf{G}})$ given by

$$
\hat{Z}(\overline{\mathbf{G}})=\frac{1}{2} \overline{\mathbf{G}} \cdot k \overline{\mathbf{G}}
$$

where $k$ is the thermal conductivity and $\overline{\mathbf{G}}=\nabla \Theta$. After invoking the Fourier relation, $\mathbf{q}=-k \overline{\mathbf{G}}$, the variation of classical thermal potential can be rewritten as

$$
\Delta \hat{Z}(\overline{\mathbf{G}})=-\mathbf{q} \cdot \Delta \overline{\mathbf{G}}
$$

By applying the definition of scalar reduction given in Appendix A, the temperature gradient can be approximated as

$$
\Delta \overline{\mathbf{G}}=\frac{1}{m} \Delta \underline{\tau} * \underline{\mathbf{X}}=\frac{1}{m} \int_{H} \underline{w}\left\langle\mathbf{x}^{\prime}-\mathbf{x}\right\rangle \Delta \underline{\tau}\left\langle\mathbf{x}^{\prime}-\mathbf{x}\right\rangle \otimes \underline{\mathbf{X}}\left\langle\mathbf{x}^{\prime}-\mathbf{x}\right\rangle d V^{\prime}
$$

in which $\Delta \underline{\tau}$ is a scalar state; thus, not requiring the dyadic, $\otimes$ operation. It reduces to

$$
\Delta \overline{\mathbf{G}}=\frac{1}{m} \int_{H} \underline{w}\left\langle\mathbf{x}^{\prime}-\mathbf{x}\right\rangle \underline{\mathbf{X}}\left\langle\mathbf{x}^{\prime}-\mathbf{x}\right\rangle \Delta \underline{\tau}\left\langle\mathbf{x}^{\prime}-\mathbf{x}\right\rangle d V^{\prime}
$$

where $\underline{w}$ is a scalar state representing the influence function, and $m$ is the scalar weighted volume.

Its substitution into Eq. (32) leads to the following, 


$$
\Delta \hat{Z}=-\frac{1}{m} \int_{H} \mathbf{q}^{T} \underline{w}\left\langle\mathbf{x}^{\prime}-\mathbf{x}\right\rangle \underline{\mathbf{X}}\left\langle\mathbf{x}^{\prime}-\mathbf{x}\right\rangle \Delta \underline{\tau}\left\langle\mathbf{x}^{\prime}-\mathbf{x}\right\rangle d V^{\prime}
$$

Assuming that the variation of the PD thermal potential, $\Delta Z$, and classical thermal potential, $\Delta \hat{Z}$ are equal, $\Delta Z=\Delta \hat{Z}$, and comparing Eq. (29) to Eq. (35), it follows that

$$
\underline{h}(\mathbf{x}, t)\left\langle\mathbf{x}^{\prime}-\mathbf{x}\right\rangle=\frac{1}{m} \mathbf{q}^{T} \underline{w}\left\langle\mathbf{x}^{\prime}-\mathbf{x}\right\rangle \underline{\mathbf{X}}\left\langle\mathbf{x}^{\prime}-\mathbf{x}\right\rangle,
$$

and this expression relates the heat flow state to the heat flux.

\section{Bond-Based Peridynamic thermal diffusion}

If it is assumed that the heat flow density associated between two material points, $\mathbf{x}$ and $\mathbf{x}^{\prime}$ is a function of the temperature difference only between these two points, then the following expression holds true,

$$
\underline{h}(\mathbf{x}, t)\left\langle\mathbf{x}^{\prime}-\mathbf{x}\right\rangle=-\underline{h}\left(\mathbf{x}^{\prime}, t\right)\left\langle\mathbf{x}-\mathbf{x}^{\prime}\right\rangle .
$$

This leads to the specialized bond-based PD thermal diffusion. In this specialized case, the heat flow density, $f_{h}\left(\mathbf{x}^{\prime}, \mathbf{x}, t\right)$ is defined as

$f_{h}\left(\mathbf{x}^{\prime}, \mathbf{x}, t\right)=\underline{h}(\mathbf{x}, t)\left\langle\mathbf{x}^{\prime}-\mathbf{x}\right\rangle-\underline{h}\left(\mathbf{x}^{\prime}, t\right)\left\langle\mathbf{x}-\mathbf{x}^{\prime}\right\rangle=2 \underline{h}(\mathbf{x}, t)\left\langle\mathbf{x}^{\prime}-\mathbf{x}\right\rangle$.

so that the PD heat conduction equation can be written as

$$
\rho c_{v} \dot{\Theta}(\mathbf{x}, t)=\int_{H} f_{h}\left(\Theta^{\prime}, \Theta, \mathbf{x}^{\prime}, \mathbf{x}, t\right) d V_{\mathbf{x}^{\prime}}+\rho s_{b}(\mathbf{x}, t) .
$$

The term, $f_{h}$, also referred to as the thermal response function, is the heat flow density function which governs the interaction of only material point $\mathbf{x}$ with $\mathbf{x}^{\prime}$. In the case of bond-based PD thermal diffusion, the pairwise interactions are independent of each other, and the heat flow between a pair of material points does not depend on the temperature difference between other pairs of material points. The thermal response function, $f_{h}\left(\mathbf{x}^{\prime}, \mathbf{x}\right)$ is zero for material points outside the horizon; i.e., $|\xi|=\left|\mathbf{x}^{\prime}-\mathbf{x}\right|>\delta$.

\section{Thermal Response Function}

The pairwise heat flow density can be related to the microthermal potential through

$$
f_{h}=\frac{\partial z}{\partial \tau} .
$$


The microthermal potential, $z$ represents the thermal potential between a pair of interacting points. The temperature difference between the material points $\mathbf{x}^{\prime}$ and $\mathbf{x}$ at any time is given by

$$
\tau\left(\mathbf{x}^{\prime}, \mathbf{x}, t\right)=\Theta\left(\mathbf{x}^{\prime}, t\right)-\Theta(\mathbf{x}, t)
$$

The thermal potential at point, $\mathbf{x}$ is then a summation over all microthermal potentials associated with this point, and is defined as,

$Z(\mathbf{x}, t)=\frac{1}{2} \int_{H} z\left(\mathbf{x}^{\prime}, \mathbf{x}, t\right) d V_{\mathbf{x}^{\prime}}$.

The pairwise heat flow density function, $f_{h}$, can be expressed as

$f_{h}\left(\mathbf{x}^{\prime}, \mathbf{x}, t\right)=\kappa \frac{\tau\left(\mathbf{x}^{\prime}, \mathbf{x}, t\right)}{|\xi|}$,

where $\kappa$ is the thermal microconductivity. The microthermal potential corresponding to the thermal response functions, $f_{h}$ can be obtained as

$$
z=\kappa \frac{\tau^{2}}{2|\xi|},
$$

The microconductivity is a PD parameter which can be related to the standard conductivity for a specified horizon. It can be determined by equating the peridynamic thermal potential to the classical thermal potential at a point arising from a simple linear temperature field a suggested by Agwai [22]. The expression for the microconductivity will differ depending on the form of the thermal response function. There are other forms as given by Bobaru and Duangpanya [18, 19] and Gerstle et al. [17].

In the most general case, heat transfer through a medium is three-dimensional. However, certain problems can be classified as two- or one dimensional depending on the relative magnitudes of heat transfer rates in different directions. Their explicit forms are derived in Appendix B as

$$
\begin{aligned}
& \kappa=\frac{2 k}{A \delta^{2}} \text { one dimensional } \\
& \kappa=\frac{6 k}{\pi h \delta^{3}} \text { two dimensional } \\
& \kappa=\frac{6 k}{\pi \delta^{4}} \text { three dimensional }
\end{aligned}
$$


These parameters are determined by computing the thermal potential of a material point whose horizon is completely embedded in the material. The value of this parameter depends on the domain of integration defined by the horizon. Therefore, the value of $\kappa$ requires correction if the material point is close to free surfaces or material interfaces (Fig. C2). The details of the correction procedure are explained in Appendix C.

\section{Initial and boundary conditions}

The PD thermal equation does not contain any spatial derivatives; thus, boundary conditions are, in general, not necessary for the solution of an integro-differential equation. However, such condition on temperature can be imposed in a "fictitious material layer" along the boundary of a nonzero volume.

Heat flux does not directly appear in the PD thermal diffusion equation. Therefore, the application of heat flux is also different from that of the classical heat conduction theory. The difference can be illustrated by considering a region, $\Omega$ that is in thermal equilibrium. If this region is fictitiously divided into two domains, $\Omega^{-}$and $\Omega^{+}$as shown in Fig. 2, there must be rate of heat flow $\dot{Q}^{+}$and $\dot{Q}^{-}$entering through the cross-sectional surfaces, $\partial \Omega$ of domain $\Omega^{+}$and $\Omega^{-}$.

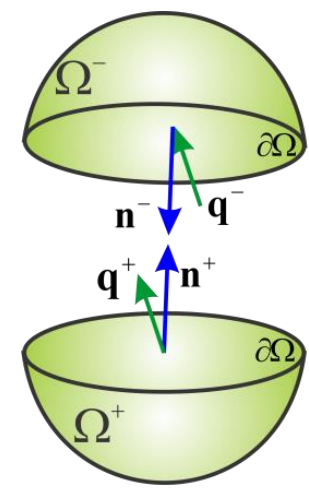

(a)
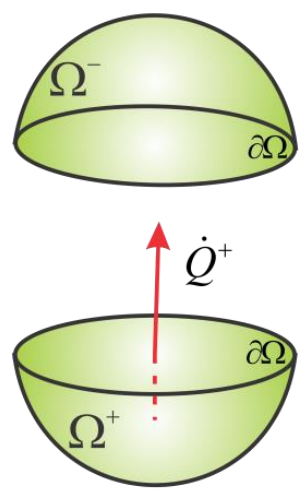

(b)

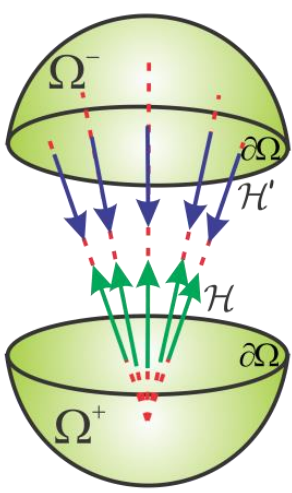

(c)

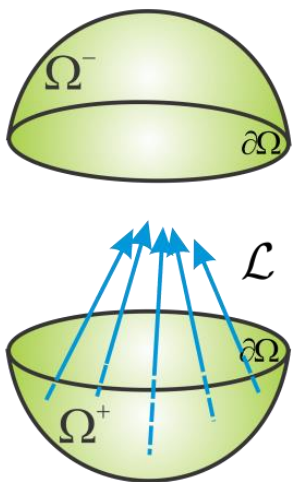

(d)

Figure 2. Boundary conditions: (a) heat fluxes through the cross sectional area (b) heat flow rate in classical heat conduction theory, (c) heat flow density of a material point in domain $\Omega^{+}$with other material points in domain $\Omega^{-}$, (d) heat flux density from domain $\Omega^{+}$due to domain $\Omega^{-}$.

According to classical heat conduction theory, the heat flow rates, $\dot{Q}^{+}$and $\dot{Q}^{-}$can be determined by integrating the normal component of the heat flux over the cross-sectional area, $\partial \Omega$, of domains $\Omega^{+}$and $\Omega^{-}$as

$\dot{Q}^{+}=-\int_{\partial \Omega} \mathbf{q}^{+} \cdot \mathbf{n}^{+} d \mathcal{S}$

and 
$\dot{Q}^{-}=-\int_{\partial \Omega} \mathbf{q}^{-} \cdot \mathbf{n}^{-} d \mathcal{S}$

in which $\mathbf{q}^{+}$and $\mathbf{q}^{-}$are the heat fluxes across the surfaces with unit normal, $\mathbf{n}^{+}$and $\mathbf{n}^{-}$ of domains $\Omega^{+}$and $\Omega^{-}$as shown in Fig $2 \mathrm{a}-\mathrm{b}$

In the case of the PD theory, the material points located in domain $\Omega^{+}$interact with the other material points in domain $\Omega^{-}$(Fig. 2c). Thus, the heat flow rate, $\dot{Q}^{+}$can be computed by volume integration of the heat flux densities (Fig. 2d) over domain $\Omega^{+}$as

$\dot{Q}^{+}=\int_{\Omega^{+}} \mathcal{L}(\mathbf{x}) d V$

in which $\mathcal{L}(\mathbf{x})$, acting on a material point in domain $\Omega^{+}$is determined by

$\mathcal{L}(x)=\int_{\Omega^{-}}\left[\underline{h}(\mathbf{x}, t)\left\langle\mathbf{x}^{\prime}-\mathbf{x}\right\rangle-\underline{h}\left(\mathbf{x}^{\prime}, t\right)\left\langle\mathbf{x}-\mathbf{x}^{\prime}\right\rangle\right] d V$

Note that if the volume $\Omega^{-}$is void, the volume integration in Eq. (47b) vanishes. Hence, the heat flux cannot be applied as a boundary condition since their volume integrations result in a zero value. Therefore, the heat flux can be applied as rate of volumetric heat generation in a "real material layer" along the boundary of a nonzero volume.

\subsection{Initial conditions}

Time integration requires the application of initial temperature values at each material point in the domain, $\mathcal{R}$ as shown in Fig. 3, and they can be specified as

$\Theta(\mathbf{x}, t=0)=\Theta^{*}(\mathbf{x})$

\subsection{Boundary conditions}

Boundary conditions can be imposed as temperature, heat flux, convection and radiation. As shown in Fig. 3, the prescribed boundary temperature is imposed in a layer of fictitious region, $\mathcal{R}_{t}$ along the boundary of the actual material surface, $\mathcal{S}_{t}$ of the actual material region, $\mathcal{R}$. Based on numerical experiments, the extent of the fictitious boundary layer to be equal to the horizon, $\delta$ in order to ensure that the prescribed temperatures sufficiently reflected in the actual material region. The prescribed heat flux, convection, and radiation are imposed in boundary layer regions, $\mathcal{R}_{f}, \mathcal{R}_{c}$ and $\mathcal{R}_{r}$, respectively, with depth, $\Delta$, along the boundary of the material region, $\mathcal{R}$ as shown in Fig. 3. 


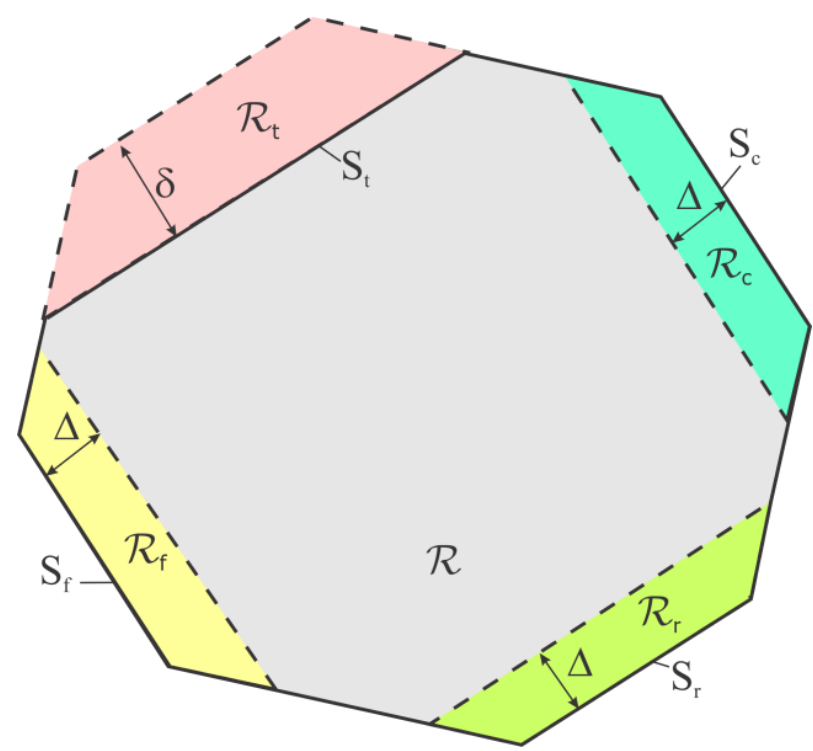

Figure 3. Boundary layers for imposing temperature, heat flux, convection and radiation

\subsubsection{Temperature}

As shown in Fig. 4a, the prescribed boundary temperature, $\Theta^{*}\left(\mathbf{x}^{*}, t\right)$ can be imposed in a layer of fictitious region, $\mathcal{R}_{t}$ along the boundary of the actual material surface, $\mathcal{S}_{t}$ as

$\Theta(\mathbf{y}, t+\Delta t)=2 \Theta^{*}\left(\mathbf{x}^{*}, t+\Delta t\right)-\Theta(\mathbf{z}, t) \quad \mathbf{x}^{*} \in \mathcal{S}_{t}, \mathbf{y} \in \mathcal{R}_{t}, \mathbf{z} \in \mathcal{R}$

in which $\mathbf{z}$ represents the position of a material point in $\mathcal{R}$, and $\mathbf{x}^{*}$ represents the location of a point on the surface, $\mathcal{S}_{t}$. Their relative position is such that the distance, $d=\left|\mathbf{x}^{*}-\mathbf{z}\right|$ between them is the shortest. The location of the image material point in $\mathcal{R}_{t}$ is obtained from $\mathbf{y}=\mathbf{z}+2 d \mathbf{n}$ with $\mathbf{n}=\left(\mathbf{x}^{*}-\mathbf{z}\right) /\left|\mathbf{x}^{*}-\mathbf{z}\right|$. The implementation of prescribed temperature boundary condition is demonstrated in Fig. 4b. For the case of $\Theta^{*}\left(\mathbf{x}^{*}, t\right)=0$, this representation enforces the temperature variation in the fictitious region to become the negative mirror image of the temperature variation near the boundary surface in the actual material as shown in Fig. 4c.

Note that the material points in the fictitious region are mirrored with respect to the tangent line of the surface. When there is a corner, the unit normal vector is assumed to be in the direction of the average of the two unit normal vectors of the associated edges, and the tangent line is assumed to be perpendicular to this vector. 


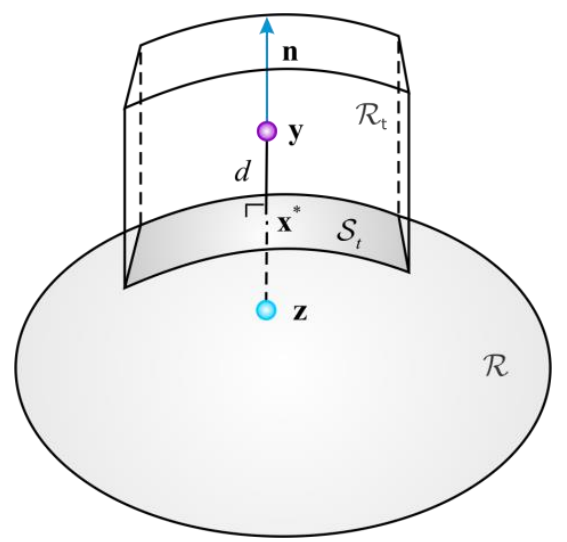

(a)

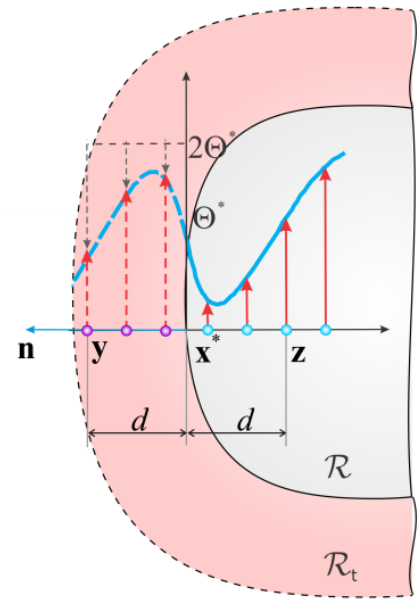

(b)

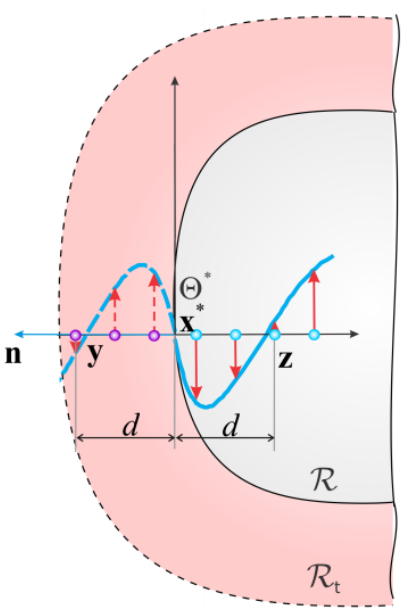

(c)

Figure 4. (a) Material point and its image in fictitious domain (b) constant temperature condition (c) zero temperature condition

\subsubsection{Heat flux}

Application of this type of boundary condition is accomplished by first calculating the rate of heat entering through the bounding surface then converting the heat flow rate, $\dot{Q}$ to a heat generation per unit volume and then specifying this volumetric heat generation to collocations points in the boundary region. Assuming the cross sectional area is constant for each material point, conversion is achieved by

$$
\tilde{Q}=\frac{\dot{Q}}{V_{f}}=\frac{-\int_{\mathcal{S}_{f}} \mathbf{q} \cdot \mathbf{n} d \mathcal{S}}{V_{f}}=-\frac{\mathbf{q} \cdot \mathbf{n} \mathcal{S}_{f}}{\mathcal{S}_{f} \Delta}=-\frac{\mathbf{q} \cdot \mathbf{n}}{\Delta}
$$

where $\tilde{Q}$ is the volumetric heat generation, $\mathbf{q}$ is the heat flux, $\mathcal{S}_{f}$ is the area over which the heat flux is applied and $V_{f}$ is the volume of the boundary region. 
In the presence of specified flux, $\mathbf{q}^{*}(\mathbf{x}, t)$ over the surface $\mathcal{S}_{f}$, shown in Fig. 3, it can be applied as the rate of volumetric heat generation in a boundary layer, $\mathcal{R}_{f}$ as

$h_{s}(\mathbf{x}, t)=-\frac{1}{\Delta} \mathbf{q}^{*}(\mathbf{x}, t) \cdot \mathbf{n} \quad$ for $\quad \mathbf{x} \in \mathcal{R}_{f}$

If there exists no specified flux, $\mathbf{q}^{*}(\mathbf{x}, t)=0$, volumetric heat generation, $\tilde{Q}$ calculated from Eq. (50) vanishes. Thus, the implementation of zero flux boundary condition can be viewed as imposing a zero valued volumetric heat generation. Alternative to this implementation, zero flux can be achieved by assigning the mirror image of the temperature values near the boundary in the actual domain to the material points in the fictitious region as shown in Fig. 5.

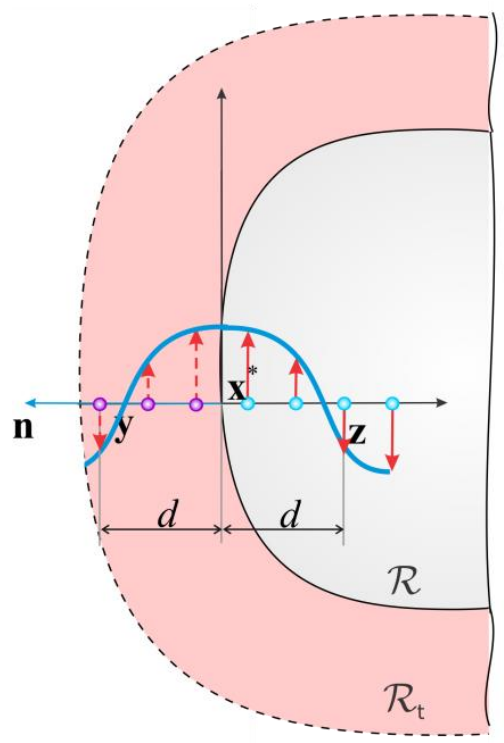

Figure 5 Material point and its image in fictitious region for imposing zero flux

\subsubsection{Convection}

Convection is a heat transfer between the surface of the body and the surrounding medium. The convection boundary condition is specified as

$\mathbf{q}(\mathbf{x}, t) \cdot \mathbf{n}=h\left(\Theta(\mathbf{x}, t)-\Theta_{\infty}\right) \quad$ for $\quad \mathbf{x} \in \mathcal{S}_{c}$

in which $\Theta_{\infty}$ is the temperature of the surrounding medium, $h$ is convective heat transfer coefficient and $\Theta(\mathbf{x}, t)$ is the temperature of the body on the surface, $\mathcal{S}_{c}$. Similar to the specified flux condition, convection can be imposed in the form of a rate of heat generation per unit volume in a boundary layer region, $\mathcal{R}_{c}$ as 
$h_{s}(\mathbf{x}, t)=\frac{1}{\Delta} h\left(\Theta_{\infty}-\Theta(\mathbf{x}, t)\right) \quad$ for $\quad \mathbf{x} \in \mathcal{R}_{c}$

\subsubsection{Radiation}

Radiation is a heat transfer between the surface of the body and the surrounding medium. The radiation boundary condition can be written as

$$
\mathbf{q}(\mathbf{x}, t) \cdot \mathbf{n}=\varepsilon \sigma\left(\Theta^{4}(\mathbf{x}, t)-\Theta_{s s}^{4}\right) \quad \text { for } \quad \mathbf{x} \in \mathcal{S}_{r}
$$

in which $\Theta_{s s}$ is the temperature of the surface surrounding the body, $\Theta(\mathbf{x}, t)$ is the surface temperature of the body, $\sigma$ is the Stefan-Boltzman constant, and $\varepsilon$ is emissivity of the boundary surface. Similar to the imposition of convection condition, radiation can also be imposed in the form of rate of heat generation per unit volume in a boundary layer region, $\mathcal{R}_{r}$ as

$$
h_{s}(\mathbf{x}, t)=\frac{1}{\Delta} \varepsilon \sigma\left(\Theta_{s s}^{4}-\Theta^{4}(\mathbf{x}, t)\right) \quad \text { for } \quad \mathbf{x} \in \mathcal{R}_{r}
$$

\section{Numerical Procedure}

Numerical techniques are employed in order to solve for the PD thermal diffusion equation. The region of interest is discretized into subdomains in which the temperature is assumed to be constant. Thus, each subdomain is represented as a single integration point located at its mass center with an associated volume. Subsequently, the integration in the governing equation, given in Eq. (39), is numerically performed as

$$
\rho_{(i)} c_{v(i)} \dot{\Theta}_{(i)}^{n}=\sum_{j=1}^{N} f_{h}\left(\tau^{n}\left(\mathbf{x}_{(j)}-\mathbf{x}_{(i)}\right)\right) V_{(j)}+h_{s(i)}^{n}
$$

for which $n$ is the time step number, $i$ represents the point of interest and $j$ represents the points within the horizon of $i$. The volume of the subdomain associated with the collocation point $\mathbf{x}_{j}$ is denoted by $V_{j}$. The time integration is accomplished using the forward difference time stepping scheme. When forward differencing is employed, the following equation is solved

$$
\Theta_{(i)}^{n+1}=\Theta_{(i)}^{n}+\frac{\Delta t}{\rho_{(i)} c_{v(i)}}\left\{\sum_{j=1}^{N} f_{h}\left(\tau^{n}\left(\mathbf{x}_{(j)}-\mathbf{x}_{(i)}\right)\right) V_{(j)}+h_{s(i)}^{n}\right\},
$$

where $\Delta t$ is the time step size. 


\subsection{Discretization and Time Stepping}

A one-dimensional region is considered to describe the details of the numerical scheme. The discretization of a one-dimensional region into subdomains is depicted in Fig. 6 . Each subdomain has one integration point. The integration point represents a material point. The solution is constructed for material point, $\mathbf{x}_{(i)}$. The material point $\mathbf{x}_{(i)}$ interacts with all points within its horizon, represented by $\mathbf{x}_{(j)}$. As shown in Fig. 6, material point $\mathbf{x}_{(i)}$ interacts with six other material points, $\mathbf{x}_{(j)}$ $(j=i-3, i-2, i-1, i+1, i+2$ and $i+3)$ in its horizon. Thus, the radius of the horizon is $\delta=3 \Delta$ where $\Delta=\left|x_{(i+1)}-x_{(i)}\right|$.

The discretized form of the PD thermal diffusion equation for material point, $\mathbf{x}_{(i)}$ becomes

$$
\rho_{(i)} c_{v(i)} \dot{\Theta}_{(i)}^{n}=\sum_{j=1}^{N} f_{h(i)(j)}^{n} V_{(j)}+h_{s(i)}^{n},
$$

in which the thermal response function, represented by $f_{h(i)(j)}^{n}$, is determined at each time step for every interaction. The discretized equation for the thermal response function, $f_{h}$ is cast as

$$
f_{h(i)(j)}^{n}=\kappa \frac{\tau_{(i)(j)}^{n}}{\left|\xi_{(i)(j)}\right|}
$$

The relative initial position is defined as $\xi_{(i)(j)}=\mathbf{x}_{(j)}-\mathbf{x}_{(i)}$, while the relative temperature is defined as $\tau_{(i)(j)}^{n}=\Theta_{(j)}^{n}-\Theta_{(i)}^{n}$. The thermal interaction of material point, $\mathbf{x}_{i}$ with the points within its horizon is illustrated in Fig.7.

The discretized thermal diffusion equation can be expanded as

$$
\begin{aligned}
\rho_{(i)} c_{v(i)} \dot{\Theta}_{(i)}^{n} & =f_{h(i)(i+1)}^{n} V_{(i+1)}+f_{h(i)(i+2)}^{n} V_{(i+2)}+f_{h(i)(i+3)}^{n} V_{(i+3)} \\
& +f_{h(i)(i-1)}^{n} V_{(i-1)}+f_{h(i)(i-2)}^{n} V_{(i-2)}+f_{h(i)(i-3)}^{n} V_{(i-3)}+h_{s(i)}^{n}
\end{aligned}
$$

For marching in time, the forward differencing scheme is used. The time derivative of temperature at material point, $\mathbf{x}_{(i)}$ is determined at the current time step, $n$ from Eq. (60). By employing time integration via the forward differencing technique, the temperature at the next time step, $(n+1)$ is determined. This algorithm may be expressed as 


$$
\Theta_{(i)}^{n+1}=\Theta_{(i)}^{n}+\Delta t \dot{\Theta}_{(i)}^{n}
$$

The forward differencing method utilized for the numerical time integration is conditionally stable. Therefore, it is necessary to develop a stability condition which sets the restriction on the time step size in order to prevent unbounded numerical solution. Similar to that performed by Silling and Askari [16], adopted a von Neumann stability analysis, and derived the stability condition as.

$$
\Delta t<\frac{\rho_{(i)} c_{v(i)}}{\sum_{j=1}^{N} \frac{\kappa}{\left|\xi_{(i)(j)}\right|} V_{(j)}} .
$$

Due to the dependence of $\kappa$ on the horizon, the stability condition given in Eq. (62) is dependent on $\delta$.

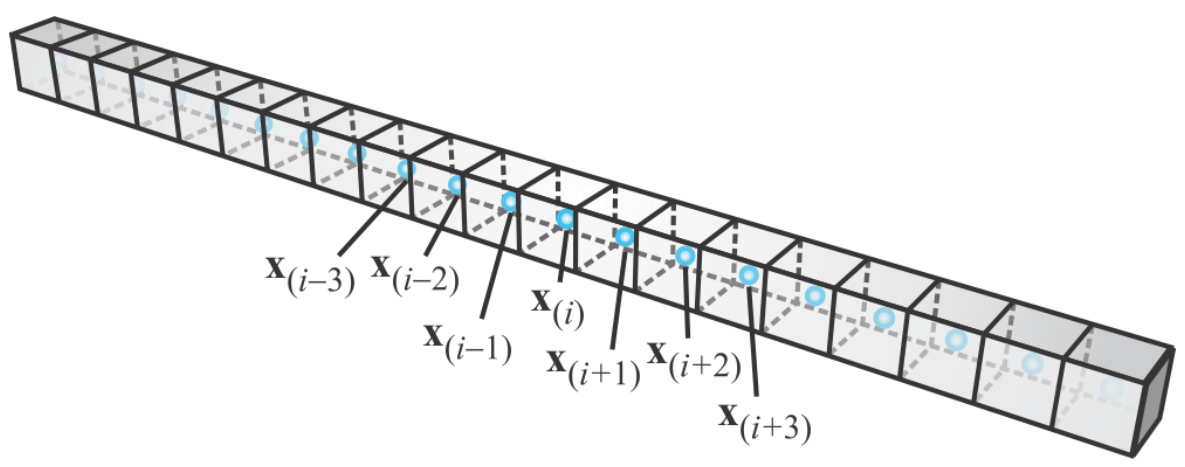

Figure 6. Discretization of one dimensional region with collocation points. 


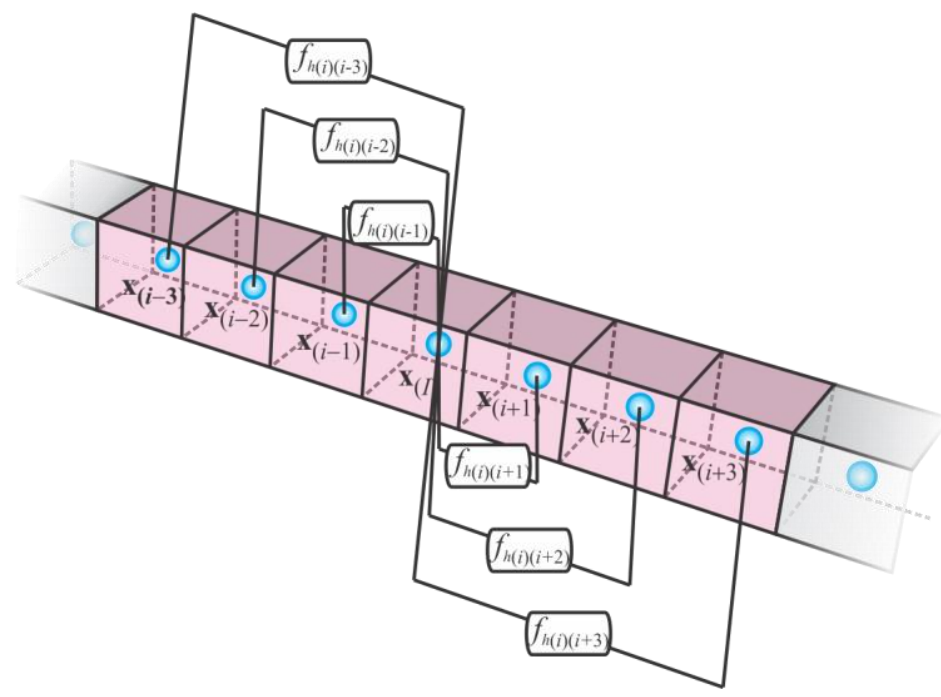

Figure 7. Thermal interaction of points with the horizon of $i$.

\section{Numerical results}

In achieving the numerical results, the bond-based peridynamics approach is adopted while utilizing the numerical schemes described in the preceding sections. The predictions from the peridynamic simulations are compared against the classical solutions to establish the validity of the peridynamic heat transfer analysis. These solutions concern a finite slab with time dependent surface temperature and convection boundary condition, plate under thermal shock with insulated boundaries, 3-D block with temperature and insulated boundaries, dissimilar materials with an insulated crack, and a thick plate with two inclined insulated cracks.

\subsection{Finite slab with time dependent surface temperature}

A finite slab initially at zero temperature is subjected to a boundary temperature that increases linearly with time whose analytical solution is available, Jiji [23]. The slab thickness is $L=0.01 \mathrm{~m}$. Its specific heat capacity, thermal conductivity and mass density are specified as $c_{v}=64 \mathrm{~J} / \mathrm{kgK}, k=233 \mathrm{~W} / \mathrm{mK}$ and $\rho=260 \mathrm{~kg} / \mathrm{m}^{3}$, respectively. It is subjected to the following initial conditions and boundary conditions:

$\Theta(x, 0)=0^{\circ} \mathrm{C} \quad 0 \leq x \leq L$

and

$\Theta(0, t)=0, \Theta(L, t)=A t$ with $A=500,0 \leq t<\infty$

As shown in Fig. 8, the spacing between material points in the PD model is $\Delta=0.0001 \mathrm{~m}$, the time step size, $\Delta t=10^{-6} \mathrm{~s}$. 
The temperature variation is predicted at $t=0.001 \mathrm{~s}, t=0.002 \mathrm{~s}, t=0.003 \mathrm{~s}$ and $t=0.004 \mathrm{~s}$. Both analytical and PD predictions are shown in Fig. 9 , and they are in close agreement. Because the temperature on the right boundary increases as a function of time, the rate of heat transfer from the right boundary also increases, as expected.

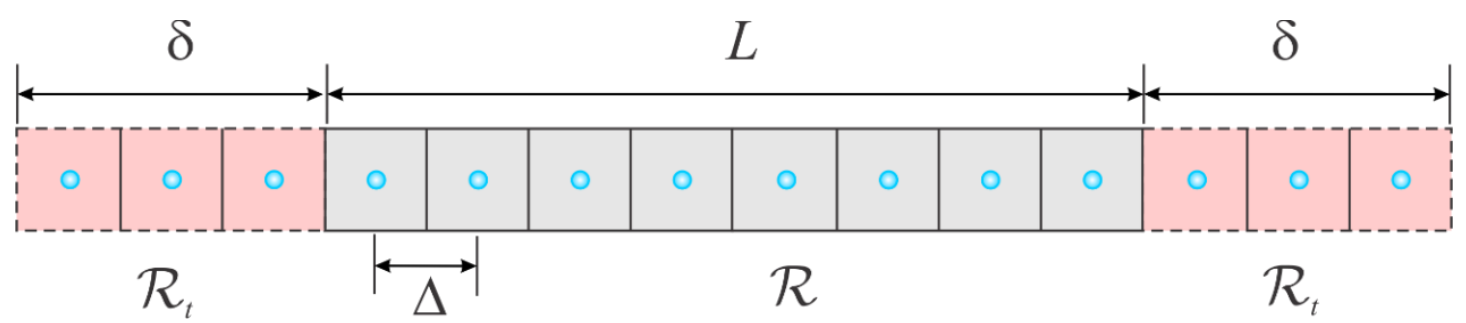

Figure 8. Discretization of the finite slab and the fictitious boundary regions for temperatures

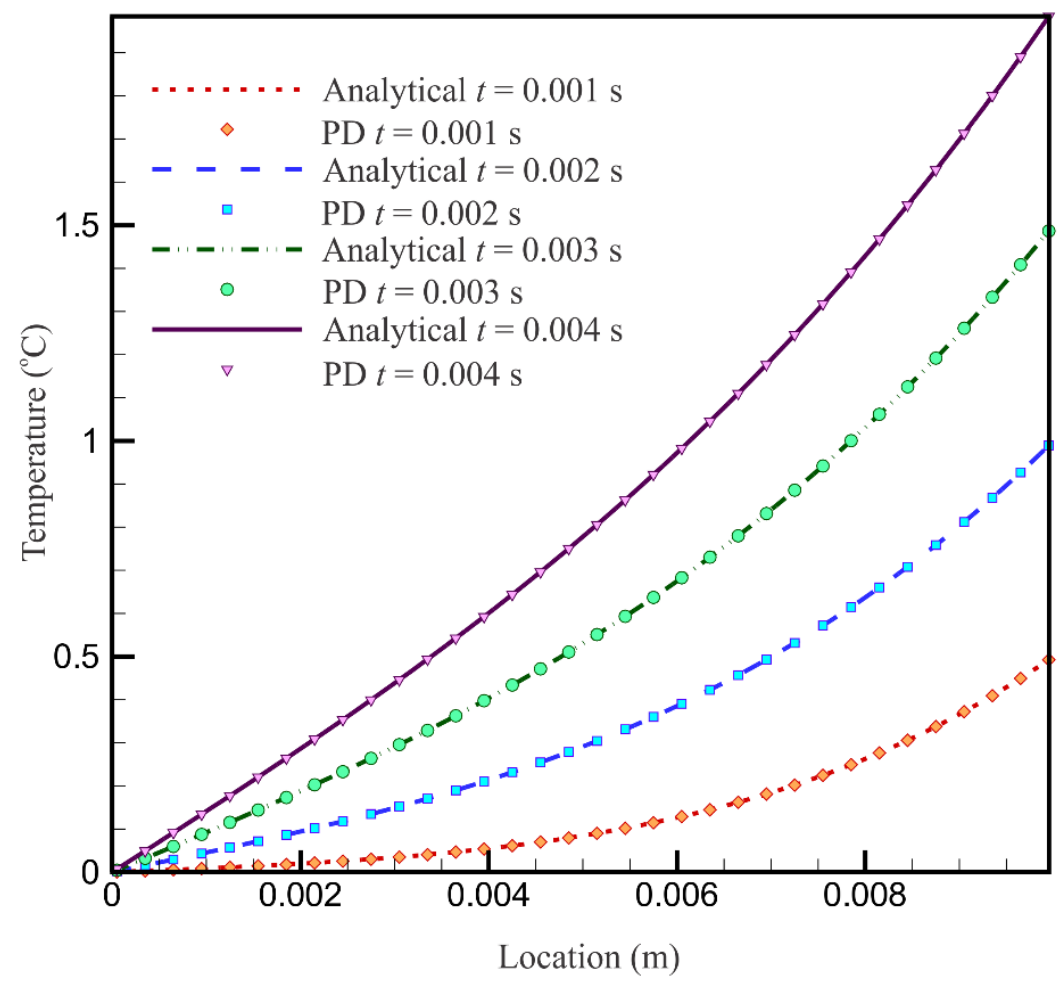

Figure 9. Temperature variations from peridynamics and classical analytical solution

\subsection{Slab with convection boundary condition}

A plate of thickness L, initially at temperature $\Theta(x, 0)=F(x)$, dissipates heat by convection for times $t>0$ from its surface into an environment at $\Theta_{\infty}=0^{\circ} \mathrm{C}$. The plate initially has a linear temperature profile, and two surfaces are subjected to convective 
heat transfer. Its analytical solution was constructed by Ozisik [10]. The slab thickness is $L=1 \mathrm{~m}$. Its specific heat capacity, thermal conductivity and mass density are specified as $c_{v}=64 \mathrm{~J} / \mathrm{kgK}, k=233 \mathrm{~W} / \mathrm{mK}$ and $\rho=260 \mathrm{~kg} / \mathrm{m}^{3}$, respectively. It is subjected to the following initial conditions and boundary conditions:

$\Theta(x, 0)=F(x), \quad 0 \leq x \leq L$, with $F(x)=x$

and

$-k \frac{\partial \Theta}{\partial x}=h_{1}\left(\Theta_{\infty}-\Theta\right), t>0$, at $x=0$

and

$k \frac{\partial \Theta}{\partial x}=h_{2}\left(\Theta_{\infty}-\Theta\right), \quad t>0$, at $x=L$

with $h_{1}=10 \mathrm{~W} / \mathrm{m}^{2} \mathrm{~K}, h_{2}=20 \mathrm{~W} / \mathrm{m}^{2} \mathrm{~K}, \Theta_{\infty}=0^{\circ} \mathrm{C}$.

As shown in Fig. 10, the spacing between material points in the PD model is $\Delta=0.002 \mathrm{~m}$, the time step size, $\Delta t=10^{-6} \mathrm{~s}$. The rates of heat generation per unit volume at $x=0$ and $x=L$ are introduced as

$h_{s 1}(\mathbf{x}, t)=\frac{1}{\Delta x} h_{1}\left(\Theta_{\infty}-\Theta(\mathbf{x}, t)\right), \mathbf{x} \in \mathcal{R}_{c 1}$

and

$h_{s 2}(\mathbf{x}, t)=\frac{1}{\Delta x} h_{2}\left(\Theta_{\infty}-\Theta(\mathbf{x}, t)\right), \mathbf{x} \in \mathcal{R}_{c 2}$

The temperature variation is predicted at $t=0.5 \mathrm{~s}, t=2.5 \mathrm{~s}, t=5 \mathrm{~s}$ and $t=10 \mathrm{~s}$. Both analytical and PD predictions are shown in Fig. 11, and they are in close agreement.

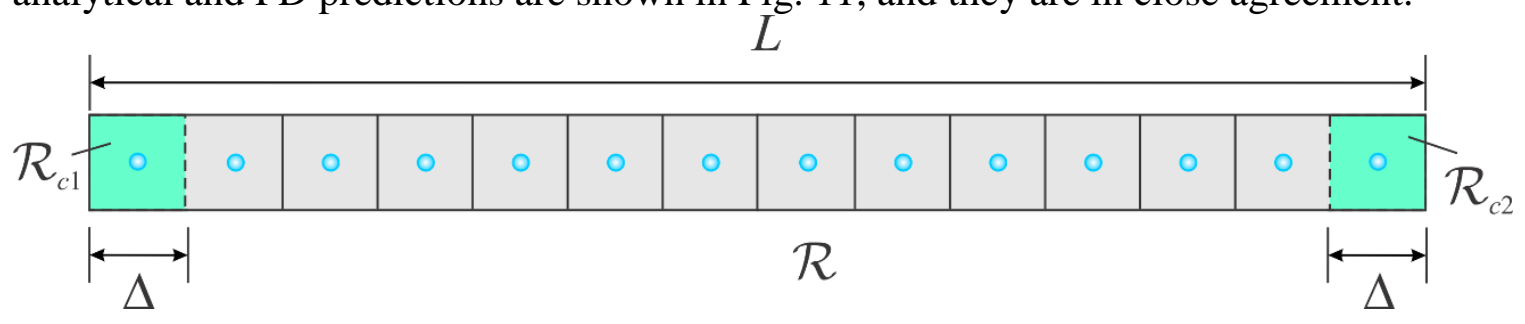

Figure 10 Discretization of the finite slab and boundary regions for convection 


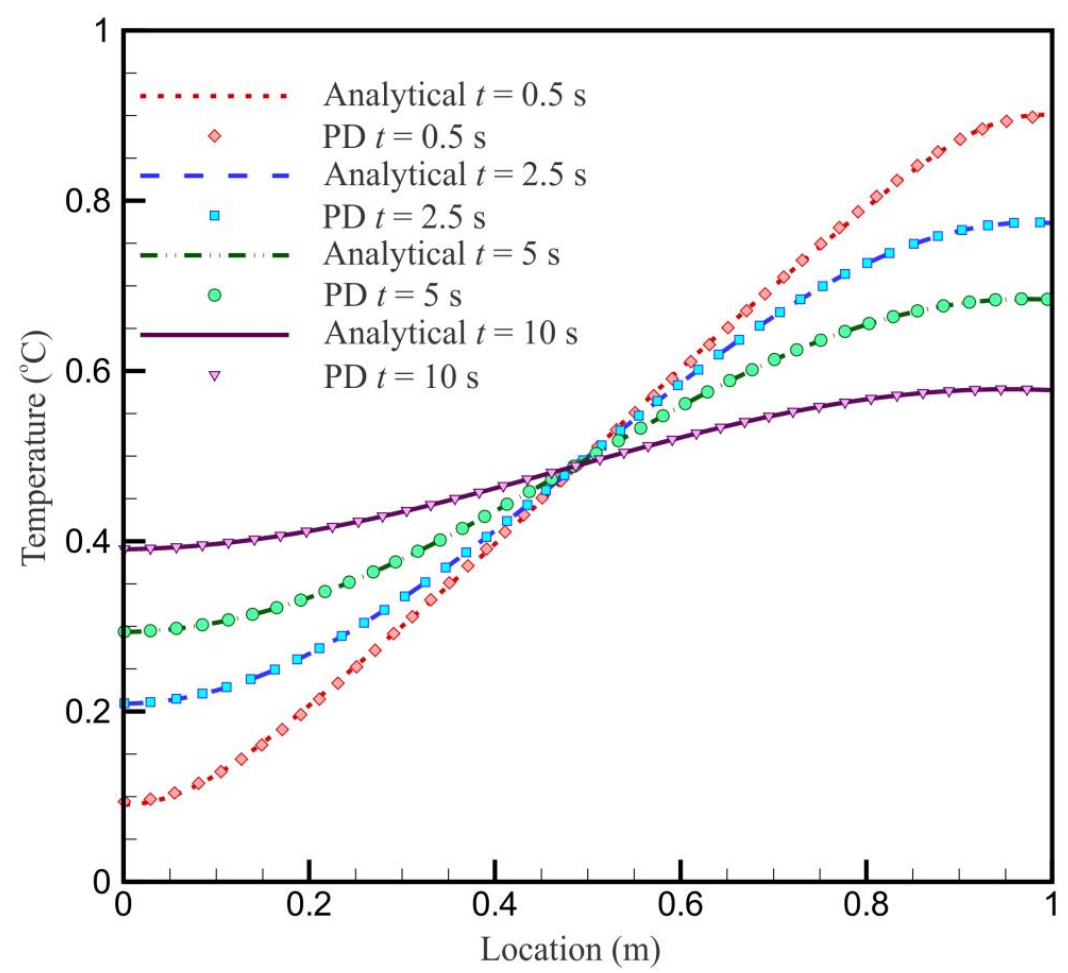

Figure 11. Temperature variations from peridynamics and classical analytical solutions 8.3 Plate under thermal shock with insulated boundaries

A square plate of isotropic material under thermal shock with insulated boundaries, shown in Fig. 12 , was first considered by Tehrani and Eslami [24] by using Boundary Element Method, (BEM). The plate has a length and width of $L=W=10 \mathrm{~m}$, and thickness of $H=1 \mathrm{~m}$. Its specific heat capacity, thermal conductivity and mass density are specified as $c_{v}=1 \mathrm{~J} / \mathrm{kgK}, k=1 \mathrm{~W} / \mathrm{mK}$ and $\rho=1 \mathrm{~kg} / \mathrm{m}^{3}$, respectively. It is subjected to the following initial conditions and boundary conditions:

$\Theta(x, y, t=0)=0^{\circ} \mathrm{C}$

and

$\Theta_{, x}(x=10, y)=0, \quad t>0$

$\Theta_{, y}(x, y= \pm 5)=0, \quad t>0$

$\Theta(x=0, t)=5 t e^{-2 t}, t>0$

As shown in Fig.12, the spacing between material points in the PD model is $\Delta=0.02 \mathrm{~m}$, the time step size, $\Delta t=5 \times 10^{-4} \mathrm{~s}$. 


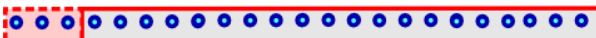

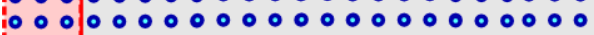
10 ० ० 10 0000000000000000000000000 10 0 ०

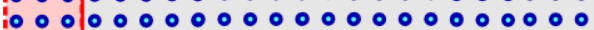
1 000000000000000000000000000 1० 00.000000000000000000000000

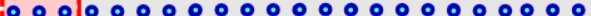
1० 000000000000000000000000000

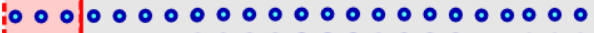
10 00000000000000000000000000

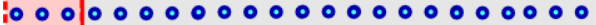
1०० 000000000000000000000 1० 0 ० 00000000000000000000000 :० $0 \circ 000000000000000000000$

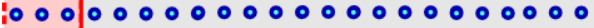

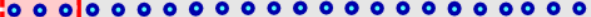

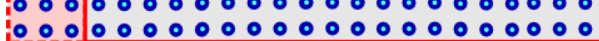

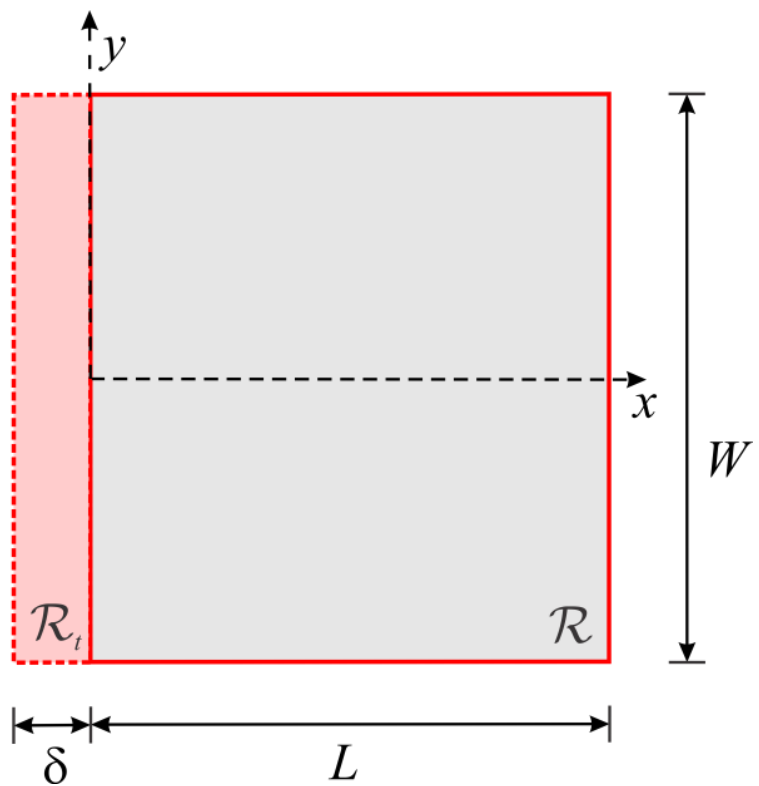

Figure 12 Peridynamic model of the plate

The temperature variations at $y=0$ are predicted for $t=3 \mathrm{~s}$ and $t=6 \mathrm{~s}$. Both BEM and PD predictions are shown in Fig.13, and they are in close agreement.

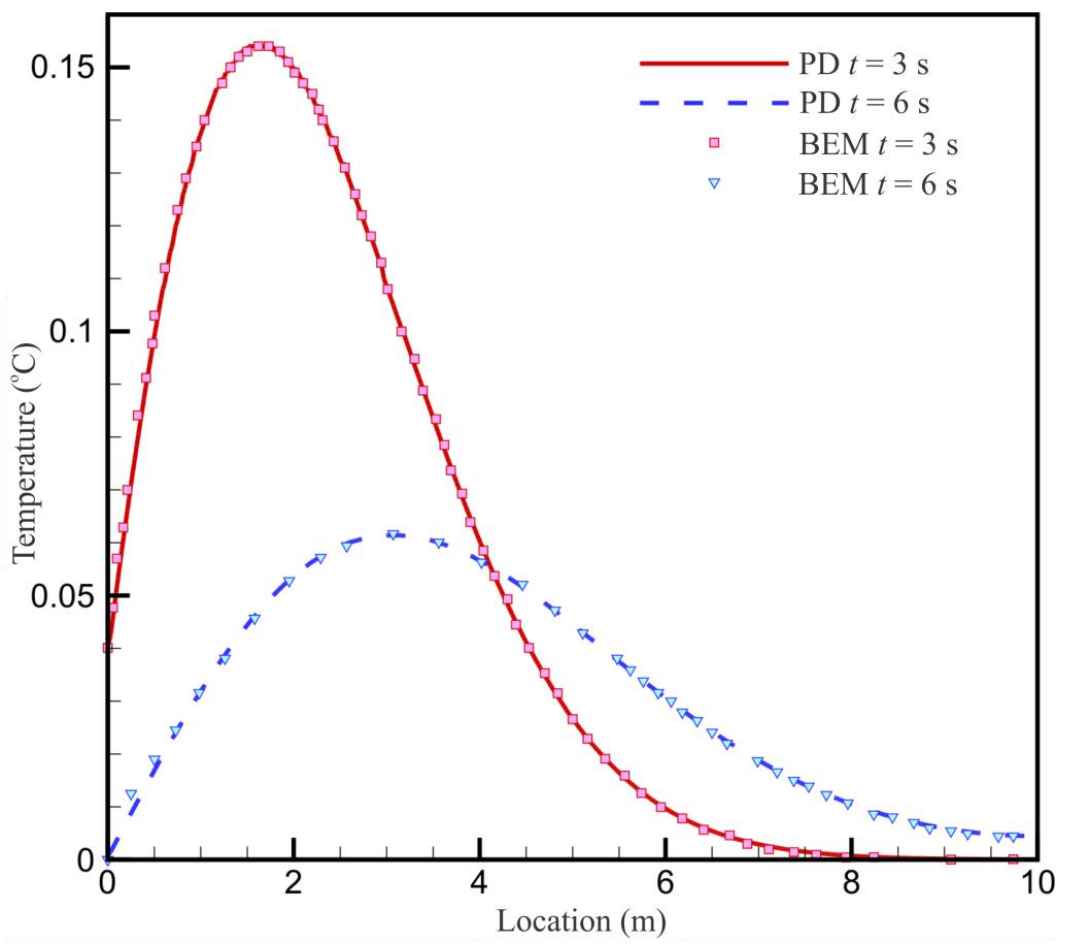

Figure 13. Temperature variation from peridynamics and BEM at $y=0$ [24 ]

\subsection{A block of material with temperature and insulated boundaries}


A block of isotropic material is subjected to constant temperatures at both ends while its lateral surfaces are insulated. The schematic of the problem is described in Fig. 14

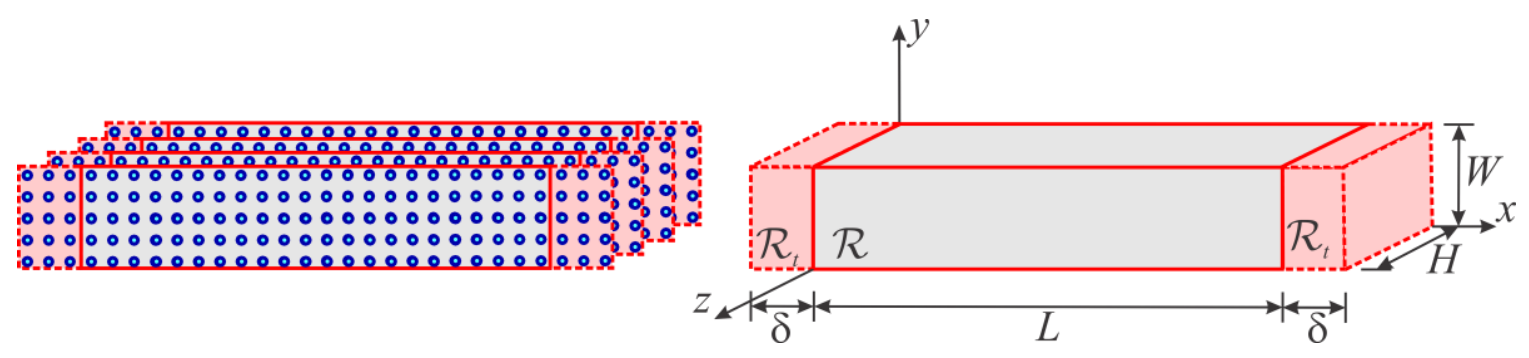

Figure 14. Peridynamic model of a three-dimensional block

The block has a length of $L=0.01 \mathrm{~m}$ and width and thickness of $W=H=0.001 \mathrm{~m}$. Its specific heat capacity, thermal conductivity and mass density are specified as $c_{v}=64 \mathrm{~J} / \mathrm{kgK}, k=233 \mathrm{~W} / \mathrm{mK}$ and $\rho=260 \mathrm{~kg} / \mathrm{m}^{3}$, respectively. It is subjected to the following initial conditions and boundary conditions:

$\Theta(x, y, z, 0)=100^{\circ} \mathrm{C}, \quad 0 \leq x \leq L, \quad 0 \leq y \leq W, \quad 0 \leq z \leq H$

and

$\Theta(0, y, z, t)=0^{\circ} \mathrm{C}, \Theta(L, y, z, t)=300^{\circ} \mathrm{C}, t>0$

$\Theta_{, y}(x, 0, z, t)=0, \Theta_{, y}(x, W, z, t)=0, t>0$

$\Theta_{, z}(x, y, 0, t)=0, \Theta_{, z}(x, y, H, t)=0, t>0$

As shown in Fig. 14, the spacing between material points in the PD model is $\Delta=0.0001 \mathrm{~m}$, the time step size, $\Delta t=10^{-7} \mathrm{~s}$. Since the block is insulated on its lateral surfaces, the temperature profile along the block can be compared with the onedimensional analytical solution given by

$$
\begin{aligned}
\Theta(x, t) & =\Theta(0, t)-\frac{\Theta(0, t)-\Theta(L, t)}{L} x-\frac{2}{L} \sum_{n=1,3,5, \ldots}^{\infty} \sin \left(\frac{n \pi}{L} x\right) \\
& \times\left[\frac{L}{n \pi}\left(\Theta(0, t)-(-1)^{n} \Theta(L, t)\right)-\frac{100 L}{n \pi}\left((-1)^{n}-1\right)\right] e^{-\frac{k}{\rho c}\left(\frac{n^{2} \pi^{2}}{L^{2}}\right) t}
\end{aligned}
$$

The temperature variation is predicted at $t=5 \times 10^{-6} \mathrm{~s}, t=5 \times 10^{-5} \mathrm{~s}, t=5 \times 10^{-4} \mathrm{~s}$, and $t=5 \times 10^{-3} \mathrm{~s}$. As the block reaches a steady-state condition, the temperature profile approaches a linear variation along the block. As observed in Fig. 15, the thermal response predicted by the peridynamic heat transfer model is in close agreement with the analytical solution. 


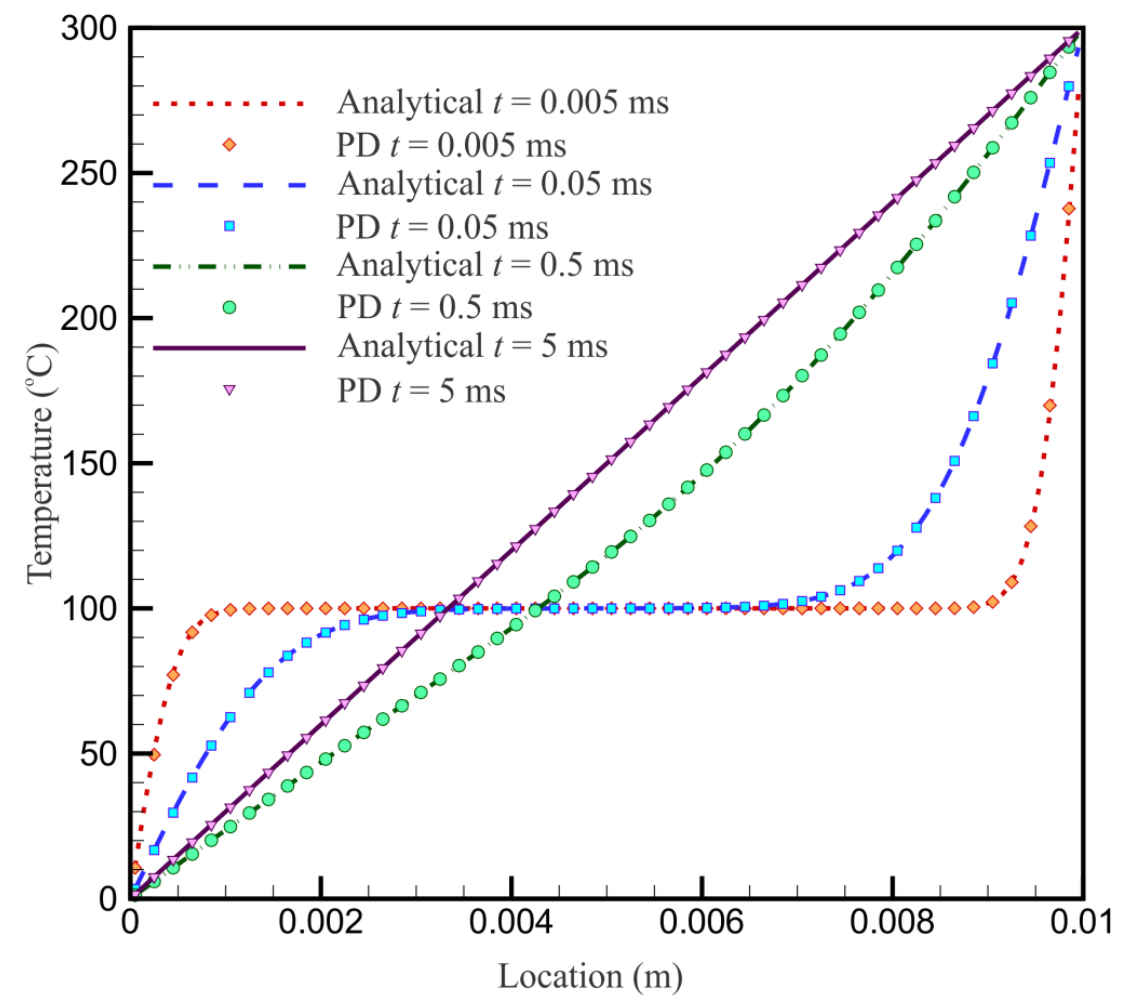

Figure 15. Temperature variations from peridynamics and classical analytical solutions 8.5 Dissimilar materials with an insulated crack

As shown in Fig. 16, a plate is made of two different materials with an insulated interface crack. The plate geometry is specified by $L=2 \mathrm{~cm}, W=2 \mathrm{~cm}, H=0.01 \mathrm{~cm}$ and $2 a=1.0 \mathrm{~cm}$. Its specific heat capacity, thermal conductivity and mass density are specified as $c_{v}=1 \mathrm{~J} / \mathrm{kgK}, k=1.14 \mathrm{~W} / \mathrm{cmK}$ and $\rho=1 \mathrm{~kg} / \mathrm{cm}^{3}$, respectively. It is subjected to the following initial conditions and boundary conditions:

$\Theta(x, y, z, 0)=0, \quad-L / 2 \leq x \leq L / 2, \quad-W / 2 \leq y \leq W / 2$

and

$\Theta(x, W / 2, t)=100^{\circ} \mathrm{C}, \Theta(x,-W / 2, t)=-100^{\circ} \mathrm{C}, t>0$

$\Theta_{, x}(L / 2, y, t)=0, \quad \Theta_{, x}(-L / 2, y, t)=0, \quad t>0$

As shown in Fig. 16, the spacing between material points in the PD model is $\Delta=0.01 \mathrm{~cm}$, the time step size, $\Delta t=10^{-4} \mathrm{~s}$. The peridynamic predictions and their comparison with ANSYS are given in Fig.17. As observed, there is a close agreement.

In order to demonstrate the three-dimensional capability of the PD analysis, the plate geometry with an insulated crack is also discretized in the thickness direction, as shown in Fig. 18. The thickness is changed to $H=0.2 \mathrm{~cm}$, and its subjected to the following initial conditions and boundary conditions: 


$$
\Theta(x, y, z, 0)=0-L / 2 \leq x \leq L / 2, \quad-W / 2 \leq y \leq W / 2, \quad-H \leq z \leq 0
$$

and

$$
\begin{aligned}
& \Theta(x, W / 2, z, t)=100^{\circ} \mathrm{C}, \quad \Theta(x,-W / 2, z, t)=-100^{\circ} \mathrm{C}, t>0 \\
& \Theta_{, x}(L / 2, y, z, t)=0, \quad \Theta_{, x}(-L / 2, y, z, t)=0, t>0 \\
& \Theta_{, z}(x, y, 0, t)=0, \quad \Theta_{, z}(x, y,-H, t)=0, t>0
\end{aligned}
$$

As shown in Fig. 18, the spacing between material points in the PD model is $\Delta=0.02 \mathrm{~cm}$, the time step size, $\Delta t=10^{-5} \mathrm{~s}$. The peridynamic results are compared with the two-dimensional predictions in the case of homogeneous plate, $k_{1}=k_{2}=k$. As observed in Fig.19, there exists a close agreement between the two- and threedimensional models.
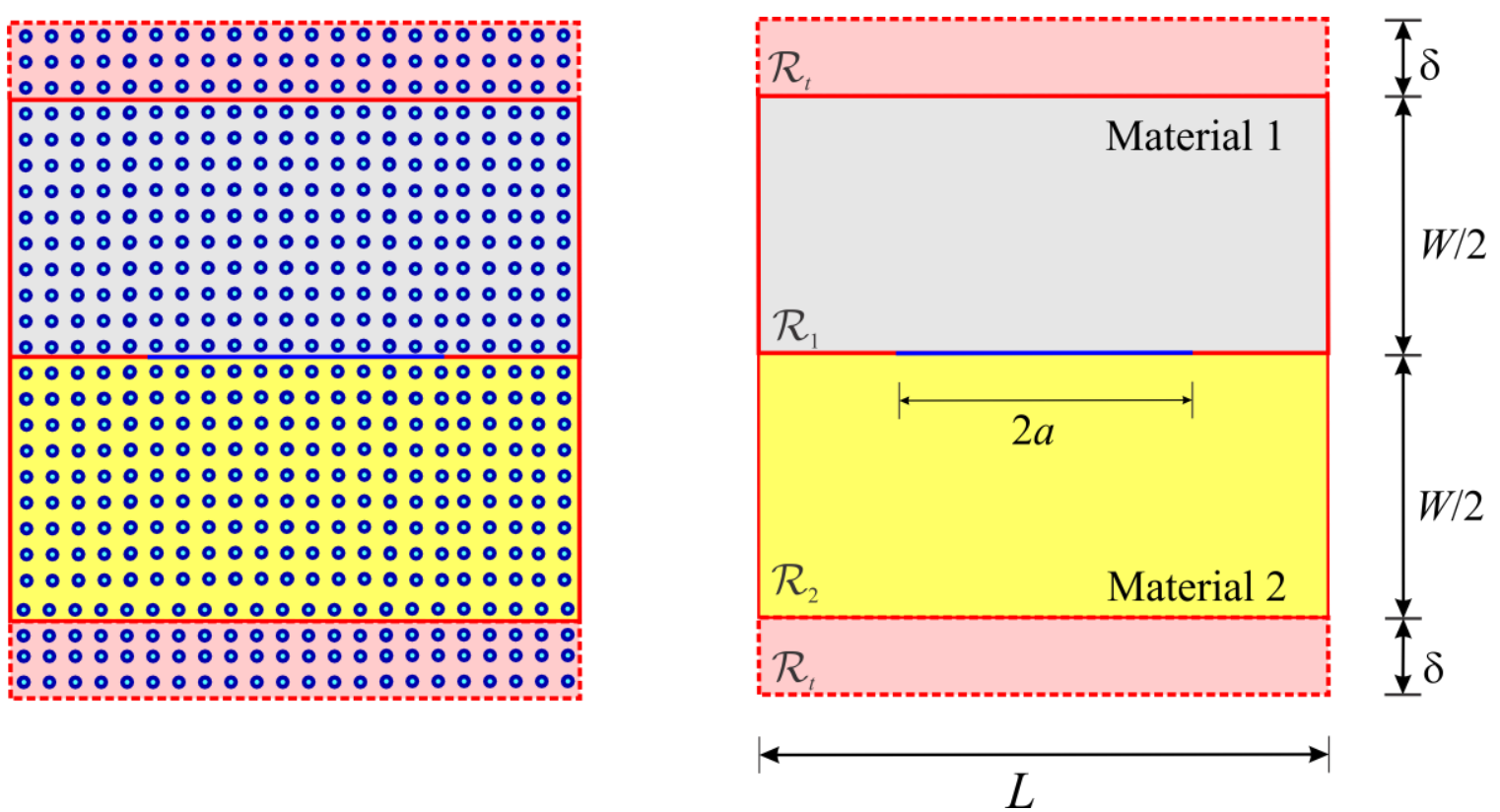

Figure 16. Peridynamic model of the plate with an insulated interface crack 


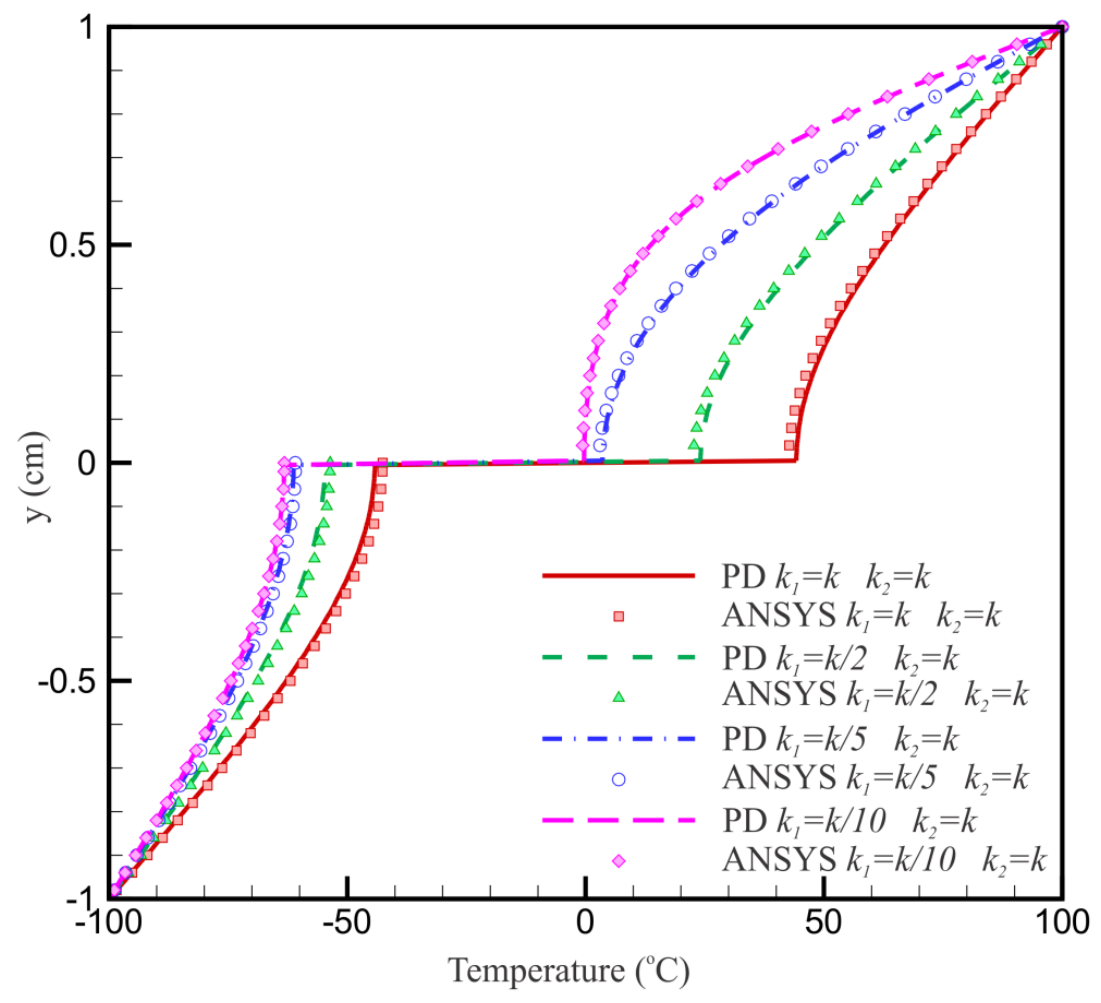

Figure 17 Temperature variations along $x=0$, across the interface of the plates with thermal conductivity $k_{1}$ for the upper half and $k_{2}$ for the lower half of the plate at

$$
t=0.5 \mathrm{~s}
$$
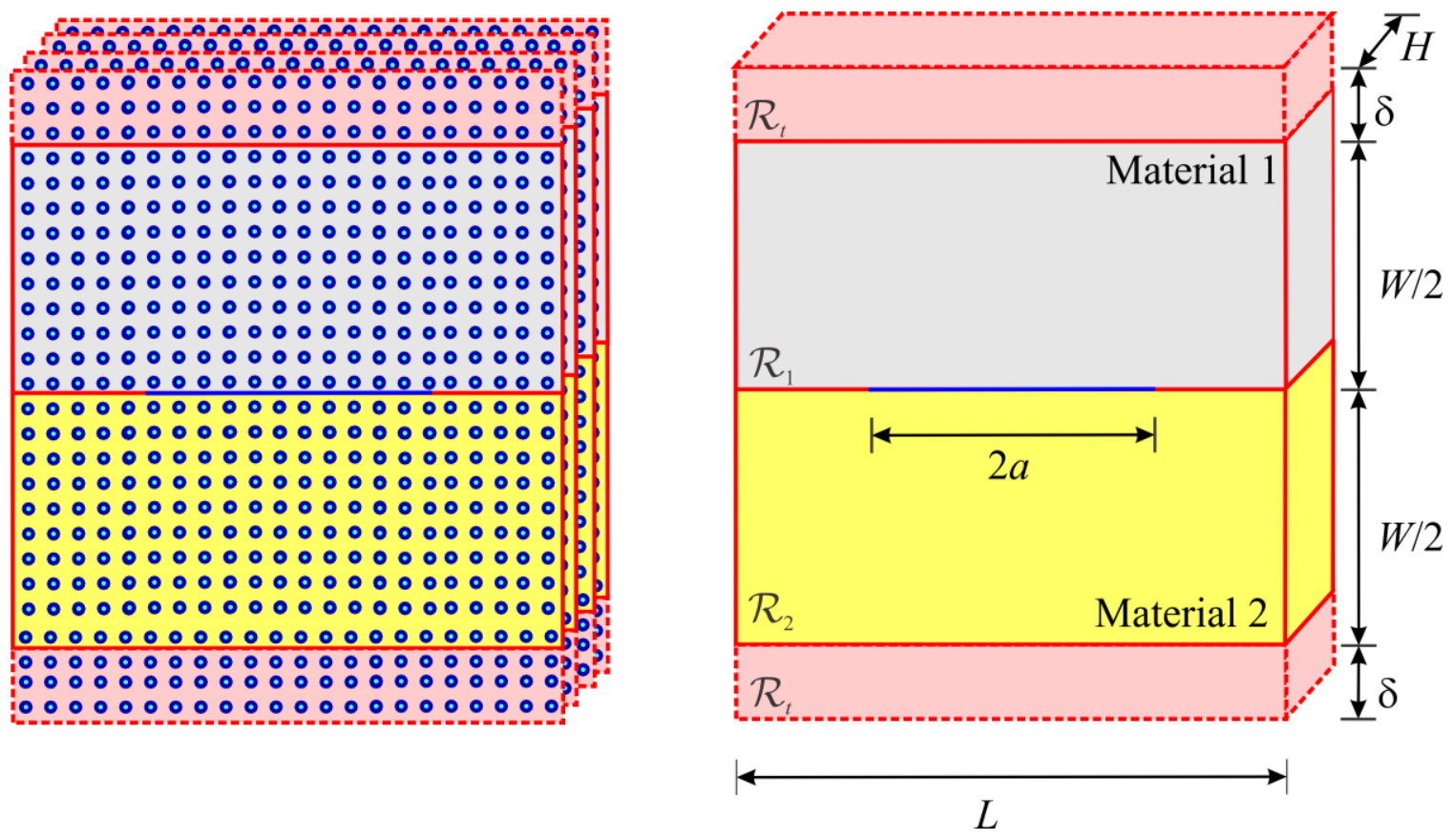

Figure 18. Three-dimensional peridynamic model of plate with a crack 


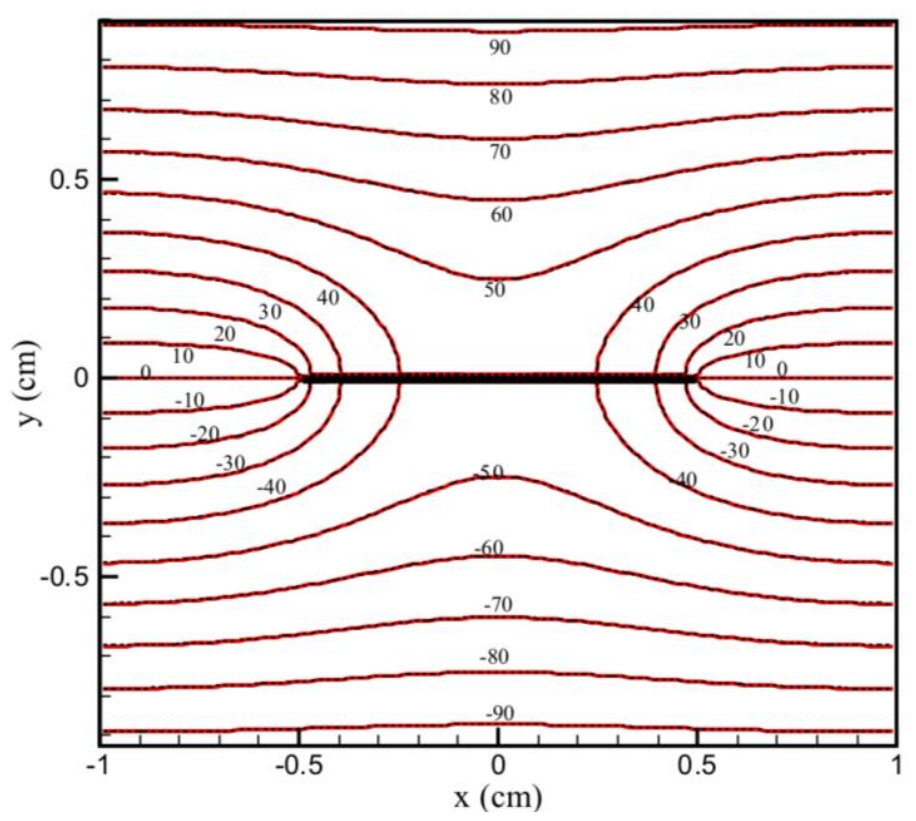

Figure 19. Temperature field from two- and three-dimensional peridynamic analysis for $k_{1}=k_{2}=k$ at $t=0.5 \mathrm{~s}$ (two-dimensional model $=$ solid line, three-dimensional model $=$ dashed line)

\subsection{Thick Plate with two inclined insulated cracks}

In order to further demonstrate the 3-D capability of the PD analysis, a thick plate with two insulated inclined cracks is considered under two different types of boundary conditions. The plate geometry is symmetric with respect to the vertical direction. For the first type of boundary conditions, the plate is subjected to constant temperature at the top and bottom surfaces while remaining surfaces are insulated. For the second type of boundary conditions, the plate is subjected to constant temperature at the top and bottom surfaces and convective heat transfer on the left and right surfaces while remaining surfaces are insulated. The discretization and PD model of the plate for these two different types of boundary conditions are shown in Fig. 20 (a) and (b).

The plate geometry is specified by $L=2 \mathrm{~cm}, W=2 \mathrm{~cm}, H=0.2 \mathrm{~cm}$ and $2 a=0.6 \mathrm{~cm}$. Crack orientations from horizontal direction are $\theta=60^{\circ}$ and $\theta=120^{\circ}$ with the distance between crack centers is $2 e=0.66 \mathrm{~cm}$. Its specific heat capacity, thermal conductivity and mass density are specified as $c_{v}=1 \mathrm{~J} / \mathrm{kgK}, k=1.14 \mathrm{~W} / \mathrm{cmK}$ and $\rho=1 \mathrm{~kg} / \mathrm{cm}^{3}$, respectively. It is has zero initial temperature, and boundary conditions are specified as:

Type -I:

$\Theta(x, W / 2, z, t)=100^{\circ} \mathrm{C}, \Theta(x,-W / 2, z, t)=-100^{\circ} \mathrm{C}, t>0$

$\Theta_{, x}(L / 2, y, z, t)=0, \Theta_{, x}(-L / 2, y, z, t)=0, t>0$

$\Theta_{, z}(x, y, 0, t)=0, \Theta_{, z}(x, y,-H, t)=0, t>0$ 
Type-II:

$\Theta(x, W / 2, z, t)=100^{\circ} \mathrm{C}, \Theta(x,-W / 2, z, t)=-100^{\circ} \mathrm{C}, t>0$

$-k T_{, x}(-L / 2, y, z, t)=h\left(\Theta_{\infty}-\Theta\right), t>0$

$k T_{, x}(L / 2, y, z, t)=h\left(\Theta_{\infty}-\Theta\right), t>0$

$h=10 \mathrm{~W} / \mathrm{cm}^{2} \mathrm{~K}, \Theta_{\infty}=0^{\circ} \mathrm{C}$

$\Theta_{, z}(x, y, 0, t)=0, \Theta_{, z}(x, y,-H, t)=0, t>0$

As shown in Fig. 20, the spacing between material points in the PD model is $\Delta=0.02 \mathrm{~m}$, the time step size, $\Delta t=10^{-5} \mathrm{~s}$. The rates of heat generation per unit volume at $x=-L / 2$ and $x=L / 2$ are introduced as

$h_{s}(\mathbf{x}, t)=\frac{1}{\Delta x} h\left(\Theta_{\infty}-\Theta(\mathbf{x}, t)\right), \mathbf{x} \in \mathcal{R}_{c}$
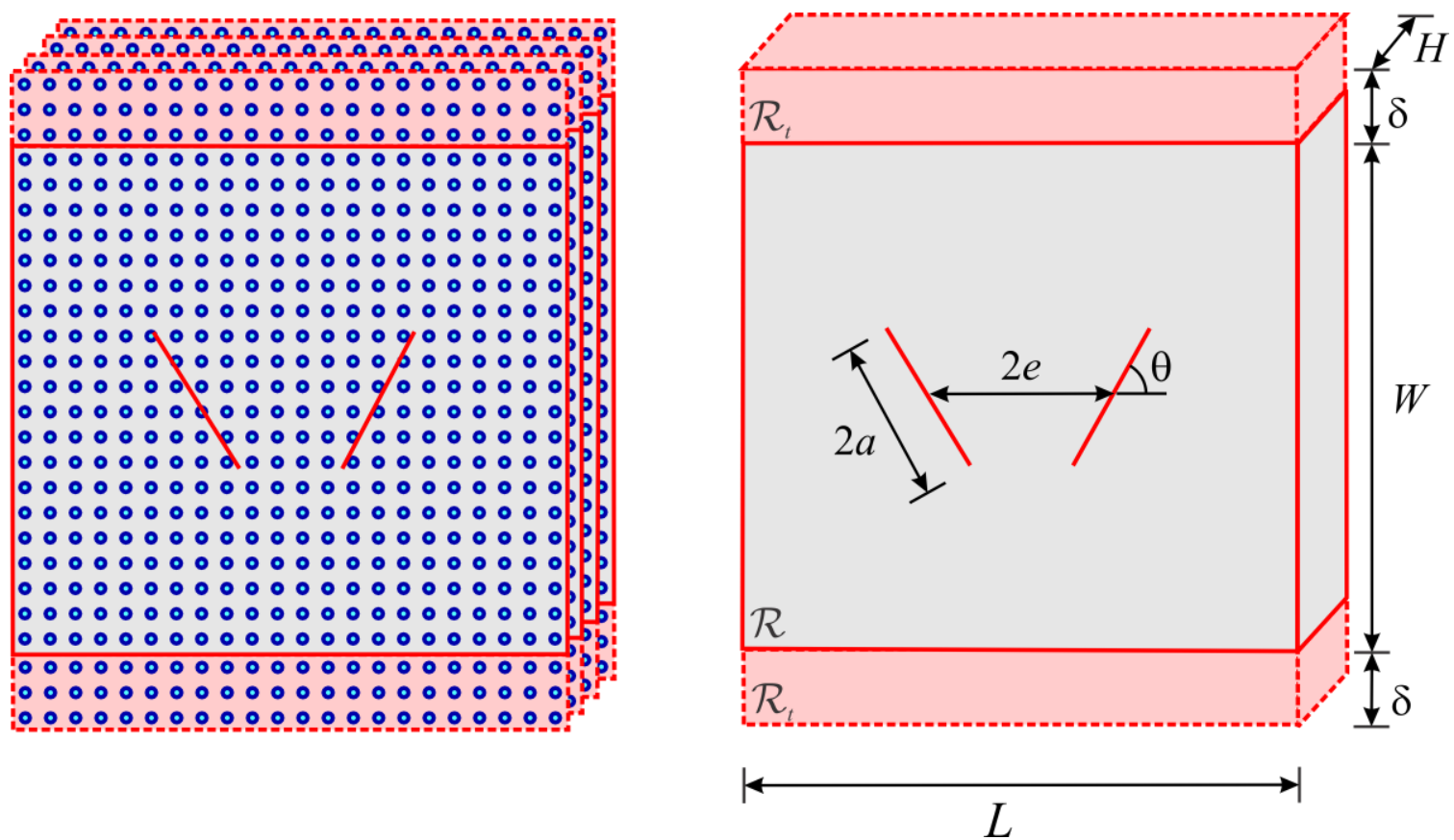

(a) 

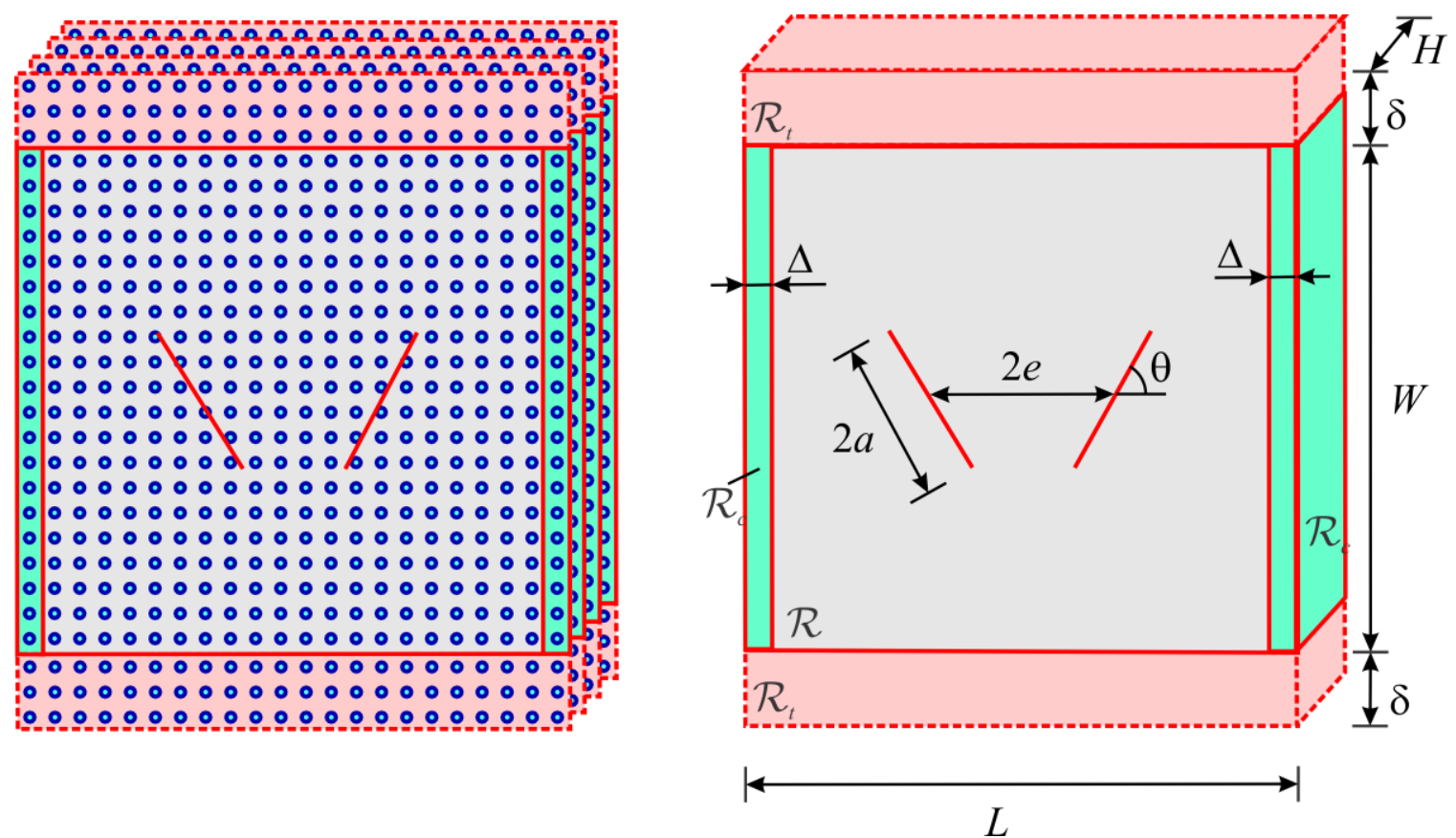

(b)

Figure 20. Peridynamic model of the thick plate: (a) boundary condition type I, and (b) boundary conditions type -II

For the first type of boundary conditions, the peridynamic prediction for the temperature field is shown in Fig. 21 . They are in close agreement with the classical solution [25, 26]. For the second type of boundary conditions, the peridynamic prediction for the temperature field is shown in Fig. 22. There exists no classical solution for comparison.

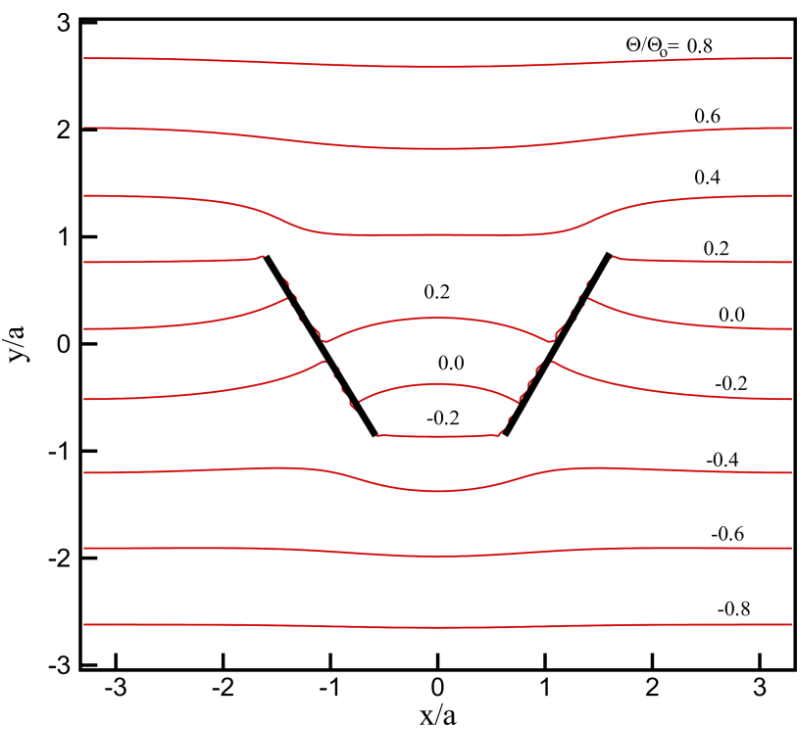

Figure 21 . Three-dimensional peridynamic temperature predictions on the midplane with a normal in $+z$ direction at $t=0.45 \mathrm{~s}$ for boundary conditions type $-\mathrm{I}$

$$
\left(\Theta_{0}=100^{\circ} \mathrm{C}\right)
$$




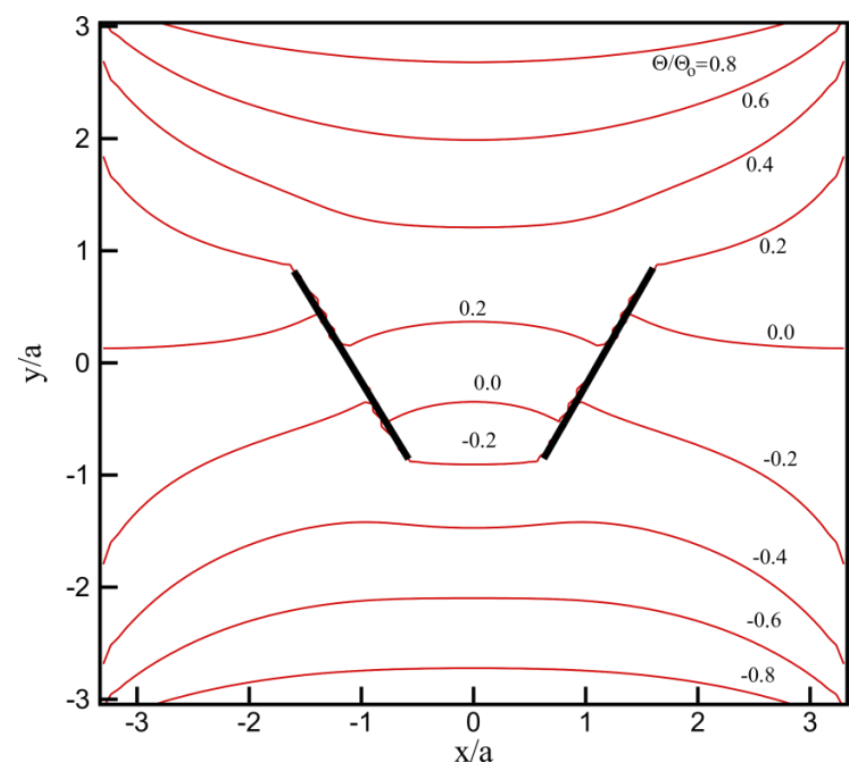

Figure 22 . Three-dimensional peridynamic temperature predictions on the midplane with a normal in $+z$ direction at $t=0.45 \mathrm{~s}$ for boundary conditions type -II

$$
\left(\Theta_{0}=100^{\circ} C\right)
$$

\section{Conclusions}

This study presents the derivation of the generalized state-based peridynamic heat transfer model based on the Lagrangian formalism, and confirmed that the governing equation represents the conservation of thermal energy. The state-based peridynamic equation was reduced to the bond-based peridynamic heat transfer equation with an approach to determine the peridynamic material parameter, the microconductivity. For all of the validation problems, the peridynamic solution was in very good agreement with the classical solution. Therefore, it is concluded that the peridynamic heat transfer model does correctly represent heat transfer. The benefit of a peridynamic heat transfer model over the classical model is the inclusion of nonlocality and the ease with which discontinuities are handled. This ground work for the peridynamic heat transfer model enables the examination of the predictive capability of the peridynamic heat transfer model in the presence of nonlocality and discontinuities.

\section{References}

[1] Tien C. L. and Chen G., "Challenges in microscale conductive and radiative heattransfer," Journal of Heat Transfer-Transactions of the ASME, Vol. 116, 1994, pp. 799807.

[2] Luciani J. F., Mora P., Virmont J., "Nonlocal heat-transport due to steep temperaturegradients," Physical Review Letters, Vol. 51, 1983, pp. 1664-1667. 
[3] Mahan G. D. and Claro F., "Nonlocal theory of thermal-conductivity," Physical Review B, Vol. 38, 1988, pp. 1963-1969.

[4] Sobolev S. L., "Equations of transfer in nonlocal media," International Journal of Heat and Mass Transfer, Vol. 37, 1994, pp. 2175-2182.

[5] Lebon G. and Grmela M., "Weakly nonlocal heat conduction in rigid solids," Physics Letters A, Vol. 214, 1996, pp. 184-188.

[6] Grmela M. and Lebon G., "Finite-speed propagation of heat: A nonlocal and nonlinear approach," Physica A, Vol. 248, 1998, pp. 428-441.

[7] Chen G., "Ballistic-diffusive equations for transient heat conduction from nano to macroscales," Journal of Heat Transfer-Transactions of the ASME, Vol. 124, 2002, pp. 320-328.

[8] Alvarez F. X. and Jou D., "Memory and nonlocal effects in heat transport: From diffusive to ballistic regimes," Applied Physics Letters, Vol. 90, 2007, pp. 083109.

[9] Tzou D. Y. and Guo Z., "Non local behavior in thermal lagging," International Journal of Thermal Sciences, Vol. 49, 2010, pp. 1133-1137.

[10] Ozisık M. N., Heat conduction, Wiley, New York: 1980.

[11] Duffey R. B. and Porthous D., "Physics of rewetting in water reactor emergency core cooling," Nuclear Engineering and Design, Vol. 25, 1973, pp. 379-394.

[12] Dorfman A. S., "Transient heat transfer between a semi-infinite hot plate and a flowing cooling liquid film," Journal of Heat Transfer-Transactions of the ASME, Vol. 126, 2004, pp. 149-154.

[13] Silling S. A., "Reformulation of elasticity theory for discontinuities and long-range forces," Journal of the Mechanics and Physics of Solids, Vol. 48, 2000, pp. 175-209.

[14] Silling S. A., Epton M., Weckner O., Xu J., Askari E., "Peridynamic states and constitutive modeling," Journal of Elasticity, Vol. 88, 2007, pp. 151-184.

[15] Silling S. A. and Lehoucq R. B., Peridynamic theory of solid mechanics. In: Advances in applied mechanics. Hassan Aref and Erik van der Giessen, editor. Elsevier 2010, pp.73-168.

[16] Silling S. A. and Askari E., "A meshfree method based on the peridynamic model of solid mechanics," Computers \& Structures, Vol. 83, 2005, pp. 1526-1535.

[17] Gerstle W., Silling S., Read D., Tewary V., Lehoucq R., "Peridynamic simulation of electromigration," CMC-Computers, Materials \& Continua, Vol. 8, 2008, pp. 75-92.

[18] Bobaru F. and Duangpanya M., "The peridynamic formulation for transient heat conduction," International Journal of Heat and Mass Transfer, Vol. 53, 2010, pp. 40474059.

[19] Bobaru F, Duangpanya M, "A peridynamic formulation for transient heat conduction in bodies with evolving discontinuities," Journal of Computational Physics, Vol. 231, 2012, pp. 2764-2785

[20] Moiseiwitsch B. L., Variational principles, Dover, Mineola (New York): 2004.

[21] Bathe K., Finite element procedures, Prentice Hall, Englewood Cliffs, N.J.: 1996.

[22] Agwai A., "A Peridynamic Approach for Coupled Fields" Ph.D. dissertation, Department of Aerospace and Mechanical Engineering, University of Arizona, 2011.

[23] Jiji M.L., Heat Conduction, Springer : 2009.

[24] Hosseini-Tehrani P., . Eslami M.R "BEM analysis of thermal and mechanical shock in a two-dimensional finite domain considering coupled thermoelasticity", Engineering Analysis with Boundary Elements, Vol. 24, 2000, pp. 249-257 
[25] C.Y. Chang, C.C. Ma, "Transient thermal conduction analysis of a rectangular plate with multiple insulated cracks by the alternating method," International Journal of Heat and Mass Transfer, Vol. 44, 2001, pp. 2423-2437.

[26] W.H. Chen, C.L. Chang, "Heat conduction analysis of a plate with multiple insulated cracks by the finite element alternating method," International Journal of Solids and Structures, Vol. 31, 1994, pp. 1343-1355.

\section{Appendix A - Concept of state}

The state concept presented by Silling et al [14] and Silling and Lehoucq [15] can be viewed as a data bank to extract information about the state of material points. For example, the vector states of reference position, $\underline{\mathbf{X}}$ and deformation, $\underline{\mathbf{Y}}$ provide information about the relative position of material points in the reference and deformed configurations. The mathematical operations for such extraction of information are denoted as

$\underline{\mathbf{X}}\left\langle\mathbf{x}^{\prime}-\mathbf{x}\right\rangle=\mathbf{x}^{\prime}-\mathbf{x}$

and

$\underline{\mathbf{Y}}\left\langle\mathbf{x}^{\prime}-\mathbf{x}\right\rangle=\mathbf{y}^{\prime}-\mathbf{y}$

in which $\mathbf{x}^{\prime}-\mathbf{x}$ and $\mathbf{y}^{\prime}-\mathbf{y}$ represent the relative position of the points $\mathbf{x}^{\prime}$ and $\mathbf{x}$ in the reference and deformed configurations. Similarly, a temperature scalar state, $\underline{\tau}$ can provide information about the temperatures, $T^{\prime}$ and $T$ at these two material points in the form

$\underline{\tau}\left\langle\mathbf{x}^{\prime}-\mathbf{x}\right\rangle=T^{\prime}-T$

The tensor product of vector states $\underline{\mathbf{A}}$ and $\underline{\mathbf{D}}$ is defined as

$$
\underline{\mathbf{A}} * \underline{\mathbf{D}}=\int_{H} \underline{w}\left\langle\mathbf{x}^{\prime}-\mathbf{x}\right\rangle \underline{\mathbf{A}}\left\langle\mathbf{x}^{\prime}-\mathbf{x}\right\rangle \otimes \underline{\mathbf{D}}\left\langle\mathbf{x}^{\prime}-\mathbf{x}\right\rangle d H
$$

where $\underline{w}$ is the influence function, a scalar state and $\otimes$ represents the dyadic product of two vectors i.e., $\mathbf{C}=\mathbf{a} \otimes \mathbf{b}$ or $C_{i j}=a_{i} b_{j}$.

The reverse transformation from a vector state to a second-order tensor, which is called the "reduction" process, can be approximated by the expression given by Silling et al. [14],. The tensor $\mathcal{R}\{\underline{\mathbf{Y}}\}$ is the vector state reduction of the vector state, $\underline{\mathbf{Y}}$ and it is defined as

$$
\mathcal{R}\{\underline{\mathbf{Y}}\}=(\underline{\mathbf{Y}} * \underline{\mathbf{X}}) \mathbf{K}^{-1} .
$$


The shape tensor, $\mathbf{K}$, is defined as

$$
\mathbf{K}=\underline{\mathbf{X}} * \underline{\mathbf{X}}
$$

Therefore, the shape tensor, $\mathbf{K}$, can be obtained as

$$
\mathbf{K}=\int_{H} \underline{w}\left\langle\mathbf{x}^{\prime}-\mathbf{x}\right\rangle \underline{\mathbf{X}}\left\langle\mathbf{x}^{\prime}-\mathbf{x}\right\rangle \otimes \underline{\mathbf{X}}\left\langle\mathbf{x}^{\prime}-\mathbf{x}\right\rangle d H .
$$

The influence function, $\underline{w}\left\langle\mathbf{x}^{\prime}-\mathbf{x}\right\rangle$, can be chosen as

$\underline{w}\left\langle\mathbf{x}^{\prime}-\mathbf{x}\right\rangle=\frac{\delta}{\left|\mathbf{x}^{\prime}-\mathbf{x}\right|}$,

with $\delta$ defining the radius of the horizon, $H$.

Based on the definition of reduction, Eq. (A.4), a scalar state, $\underline{a}$ can be reduced to a vector, $\mathcal{R}\{\underline{a}\}$ as

$$
\mathcal{R}\{\underline{a}\}=(\underline{a} * \underline{\mathbf{X}}) m^{-1}
$$

Hence, a vector state, $\underline{a}$ can be reduced to a vector, $\mathbf{f}$ as

$$
\mathbf{f}=\mathcal{R}\{\underline{a}\}
$$

The scalar weighted volume, $m$ is defined as

$$
m=\int_{H} \underline{w}\left\langle\mathbf{x}^{\prime}-\mathbf{x}\right\rangle|\underline{\mathbf{X}}|\left\langle\mathbf{x}^{\prime}-\mathbf{x}\right\rangle \otimes|\underline{\mathbf{X}}|\left\langle\mathbf{x}^{\prime}-\mathbf{x}\right\rangle d H
$$

The dyadic, $\otimes$, operation annuls because both $\underline{a}\left\langle\mathbf{x}^{\prime}-\mathbf{x}\right\rangle$ and $|\underline{\mathbf{X}}|\left\langle\mathbf{x}^{\prime}-\mathbf{x}\right\rangle=\left|\mathbf{x}^{\prime}-\mathbf{x}\right|$ are scalar; thus, the reduction expression can be rewritten as

$\mathbf{f}=\frac{1}{m} \int_{H} \underline{w}\left\langle\mathbf{x}^{\prime}-\mathbf{x}\right\rangle \underline{\mathbf{X}}\left\langle\mathbf{x}^{\prime}-\mathbf{x}\right\rangle \underline{a}\left\langle\mathbf{x}^{\prime}-\mathbf{x}\right\rangle d H$,

with

$$
m=\int_{H} \underline{w}\left\langle\mathbf{x}^{\prime}-\mathbf{x}\right\rangle\left|\mathbf{x}^{\prime}-\mathbf{x}\right|\left|\mathbf{x}^{\prime}-\mathbf{x}\right| d H,
$$


Substituting for the influence function, $\underline{w}\left\langle\mathbf{x}^{\prime}-\mathbf{x}\right\rangle$ from Eq. (A.7), scalar weighted volume can be evaluated as

$$
m=\delta \int_{H}\left|\mathbf{x}^{\prime}-\mathbf{x}\right| d H
$$

In light of Fig. A.1, it can be explicitly evaluated as

$$
m=\delta \int_{0}^{\delta} \int_{0}^{2 \pi} \int_{0}^{\pi} \xi \xi^{2} \sin (\phi) d \phi d \theta d \xi=\frac{3}{4} V \delta^{2}
$$

The scalar weighted volume can be viewed as a quantity that serves as volume averaging of the product of a scalar and vector states, $\underline{a} * \underline{\mathbf{X}}$.

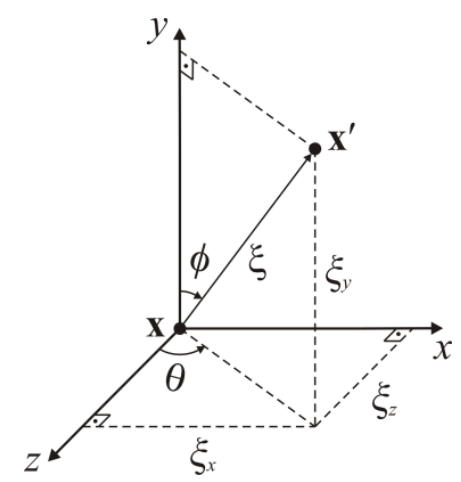

Figure A.1. Components of the position vector, $\xi$, between material points at $\mathbf{x}$ and $\mathbf{x}^{\prime}$.

\section{Appendix B - Peridynamic microconductivity}

The microconductivity can be determined by equating the peridynamic thermal potential to the classical thermal potential at a point arising from a simple linear temperature field.

\section{One-dimensional analysis}

For one-dimensional analysis, a simple linear temperature field of the form, $\Theta(x)=x$ results in the PD temperature difference of

$$
\tau=\Theta\left(x^{\prime}\right)-\Theta(x)=x^{\prime}-x=\xi
$$

Invoking this temperature difference into Eq. (44) results in the PD microthermal potential as

$$
z=\kappa \frac{\xi^{2}}{2|\xi|}
$$


where $|\xi|=\left|x^{\prime}-x\right|$. Substituting for $z$ from Eq. (B.2) into Eq. (42) and performing the integration leads to PD thermal potential as

$Z=\frac{1}{2} \int_{H} z(\xi) d V_{\xi}=\frac{\kappa}{2} \int_{0}^{\delta}\left(\frac{\xi^{2}}{|\xi|}\right) A d \xi=\frac{\kappa \delta^{2} A}{4}$

where $A$ is the cross-sectional area of the volume associated with the material point $\mathbf{x}^{\prime}$. The corresponding classical thermal potential from Eq. (31b) is obtained as

$\hat{Z}=\frac{1}{2} k$

Equating the peridynamic thermal potential in Eq. (B.3) to the classical thermal potential given in Eq. (B.4) and solving for $\kappa$ result in the PD microconductivity for onedimensional analysis as

$\kappa=\frac{2 k}{A \delta^{2}}$

\section{Two-dimensional analysis}

For two-dimensional analysis, a simple linear temperature field of the form, $\Theta(x, y)=(x+y)$ results in the PD temperature difference of

$\tau=\Theta\left(x^{\prime}, y^{\prime}\right)-\Theta(x, y)=x^{\prime}+y^{\prime}$

for the material point of interest, $\mathbf{x}$ located at the origin $(x=0, y=0)$ Invoking this temperature difference into Eq. (44) results in the PD microthermal potential as

$z=\kappa \frac{\left(x^{\prime}+y^{\prime}\right)^{2}}{2|\xi|}$

where $|\xi|=\sqrt{x^{\prime 2}+y^{\prime 2}}$. Substituting for $z$ from Eq. (B7) into Eq. (42) and performing the integration over the horizon leads to PD thermal potential as

$Z(\mathbf{x}, t)=\frac{1}{2} \int_{0}^{2 \pi} \int_{0}^{\delta} \kappa \frac{(\xi \operatorname{Cos}(\theta)+\xi \operatorname{Sin}(\theta))^{2}}{2|\xi|} h \xi d \xi d \theta=\frac{\pi h \kappa \delta^{3}}{6}$

in which polar coordinates, $(\xi, \theta)$ are utilized to perform the integration over a disk with thickness, $h$ and radius, $\delta$. The corresponding classical thermal potential from Eq. (31b) is obtained as 
$\hat{Z}=k$

Equating the PD thermal potential in Eq. (B8) to the classical thermal potential given in Eq. (B9) and solving for $\kappa$ result in the PD microconductivity for two-dimensional analysis as

$\kappa=\frac{6 k}{\pi h \delta^{3}}$

\section{Three-dimensional analysis}

For three-dimensional analysis, a simple linear temperature field of the form, $\Theta(x, y)=(x+y+z)$ results in the PD temperature difference of

$\tau=\Theta\left(x^{\prime}, y^{\prime}, z^{\prime}\right)-\Theta(x, y, z)=\left(x^{\prime}+y^{\prime}+z^{\prime}\right)$

for the material point of interest, $\mathbf{x}$ located at the origin $(x=0, y=0, z=0)$ Invoking this temperature difference into Eq. (44) results in the PD microthermal potential as

$z=\kappa \frac{\left(x^{\prime}+y^{\prime}+z^{\prime}\right)^{2}}{2|\xi|}$

where $|\xi|=\sqrt{x^{\prime 2}+y^{\prime 2}+z^{\prime 2}}$. Substituting for $z$ from Eq. (B12) into Eq. (42) and performing the integration over the horizon leads to PD thermal potential as

$$
\begin{aligned}
Z(\mathbf{x}, t) & =\frac{1}{2} \int_{0}^{\delta} \int_{0}^{2 \pi} \int_{0}^{\pi} \kappa \frac{(\xi \operatorname{Cos}(\theta) \operatorname{Sin}(\phi)+\xi \operatorname{Sin}(\theta) \operatorname{Sin}(\phi)+\xi \operatorname{Cos}(\phi))^{2}}{2|\xi|} \operatorname{Sin} \phi d \phi d \theta \xi^{2} d \xi \\
& =\frac{\pi \kappa \delta^{4}}{4}
\end{aligned}
$$

in which spherical coordinates, $(\xi, \theta, \phi)$ are utilized to perform the integration over a sphere with radius, $\delta$. The corresponding classical thermal potential from Eq. (31b) is obtained as

$\hat{Z}=\frac{3}{2} k$

Equating the peridynamic thermal potential in Eq. (B13) to the classical thermal potential given in Eq. (B14) and solving for $\kappa$ result in the PD microconductivity for threedimensional analysis as 
$\kappa=\frac{6 k}{\pi \delta^{4}}$

\section{Appendix C - Surface effects}

Since the presence of free surfaces is problem dependent, it is impractical to resolve this issue analytically. The correction of the material parameters is achieved by numerically integrating the PD thermal potential at each material point inside the body for simple temperature distribution and comparing to its counterpart obtained from classical thermal potential.

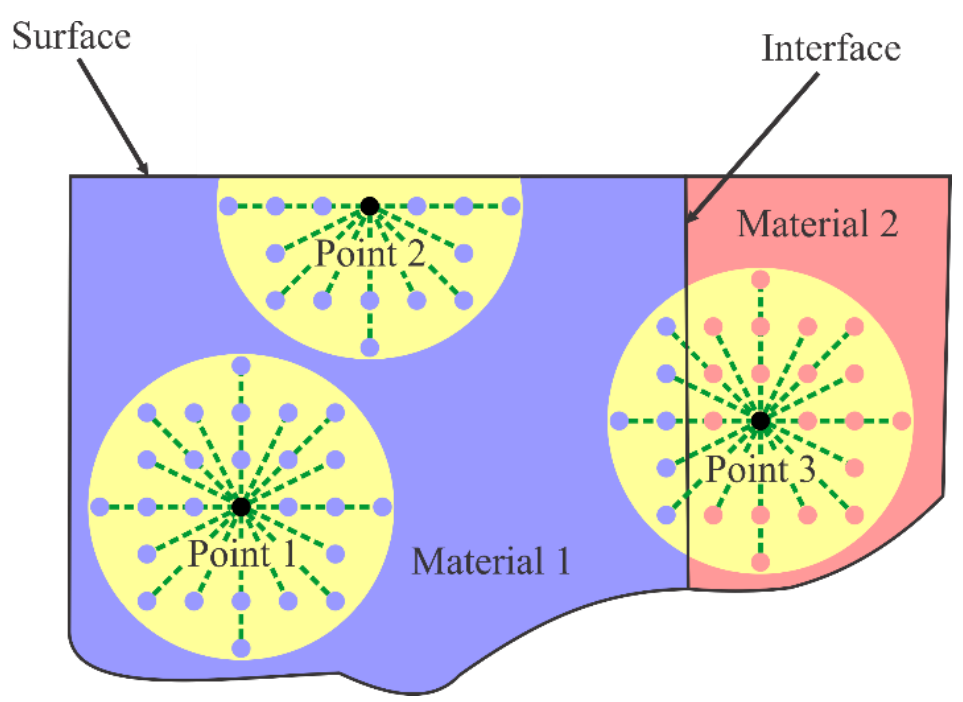

Figure C.1. Surface effects in the domain of interest.

The simple temperature distribution can be linear in form, and the corresponding thermal potential, $Z_{\infty}$ of a point completely embedded in the material is calculated using Eq. (31b). Subsequently, the PD thermal potential due to the applied linear temperature distribution is computed for each material point through numerical integration over its horizon from

$Z_{(i)}=\frac{1}{2} \int_{H} z(\xi) d V_{\xi}=\frac{1}{2} \sum_{j=1}^{N} z_{(i)(j)} V_{(j)}$

in which the micropotential, $z_{(i)(j)}$ between material points, $\mathbf{x}_{(i)}$ and $\mathbf{x}_{(j)}$ depends on the material microconductivity.

As shown in Fig. C.2, the material point, $\mathbf{x}_{(i)}$ may interact with material points $\mathbf{x}_{(j)}$ and $\mathbf{x}_{(m)}$. Material points $\mathbf{x}_{(i)}$ and $\mathbf{x}_{(j)}$ are embedded in material 1, and $\mathbf{x}_{(m)}$ is embedded in material 2. Thus, the microconductivity between points $\mathbf{x}_{(i)}$ and $\mathbf{x}_{(j)}$ is $\kappa_{(i)(j)}$, and it 
differs from $\kappa_{(i)(m)}$ between material points $\mathbf{x}_{(i)}$ and $\mathbf{x}_{(m)}$. Because the material points $\mathbf{x}_{(i)}$ and $\mathbf{x}_{(m)}$ are embedded in two different materials, their microconductivity, $\kappa_{(i)(m)}$ can be expressed in terms of an equivalent thermal conductivity as

$$
k_{(i)(m)}=\frac{\ell_{1}+\ell_{2}}{\frac{\ell_{1}}{k_{1}}+\frac{\ell_{2}}{k_{2}}}
$$

in which $\ell_{1}$ represents the segment of the distance between material points $\mathbf{x}_{(i)}$ and $\mathbf{x}_{(m)}$ in material 1 whose thermal conductivity is $k_{1}$, and $\ell_{2}$ represents the segment in material 2 whose thermal conductivity is $k_{2}$.

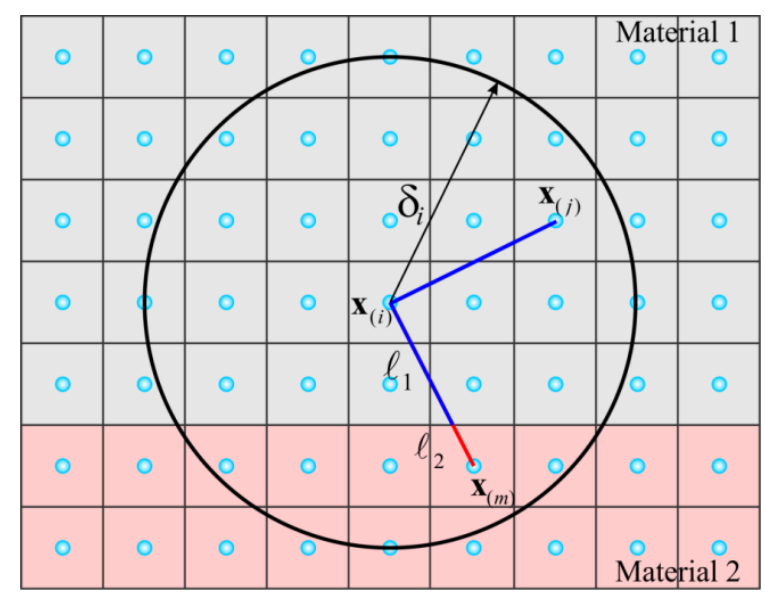

Figure C.2 Material point $\mathbf{x}_{(i)}$ close to an interface

The thermal potential of material point, $\mathbf{x}_{(i)}$ is denoted by $Z_{(i)}$. The correction factor is determined for each material point in the domain as

$g_{(i)}=\frac{Z_{\infty}}{Z_{(i)}}$

Therefore, the discretized thermal diffusion equation including the correction factor for point $\mathbf{x}_{(i)}$ becomes

$\rho_{(i)} c_{v(i)} \dot{\Theta}_{(i)}^{n}=\sum_{j=1}^{N} g_{(i)(j)} f_{h(i)(j)}^{n} V_{(j)}+\rho_{(i)} s_{b(i)}^{n}$,

where $g_{(i)(j)}=g_{(i)}+g_{(j)} / 2$. Finally, the discretized equation of motion for material point $\mathbf{x}_{(i)}$, including surface and volume correction, $v_{c}$, is rewritten as 


$$
\rho_{(i)} c_{v(i)} \dot{\Theta}_{(i)}^{n}=\sum_{j=1}^{N} g_{(i)(j)} f_{h(i)(j)}^{n}\left(v_{c(j)} V_{(j)}\right)+\rho_{(i)} s_{b(i)}^{n} .
$$

Also, the thermal response functions between material points $\mathbf{x}_{(i)}$ and $\mathbf{x}_{(j)}$ and $\mathbf{x}_{(i)}$ and $\mathbf{x}_{(m)}$ are modified to reflect the change in microconductivity as

$$
f_{h(i)(m)}^{n}=\kappa_{(i)(m)} \frac{\tau_{(i)(m)}^{n}}{\left|\xi_{(i)(m)}\right|} \quad \text { and } f_{h(i)(j)}^{n}=\kappa_{(i)(j)} \frac{\tau_{(i)(j)}^{n}}{\left|\xi_{(i)(j)}\right|}
$$




\section{FIGURE CAPTIONS}

Fig. 1 Peridynamic material points and their interaction with each other

Fig. 2 Boundary conditions: (a) heat fluxes through the cross-sectional area, (b) heat flow rate in classical heat conduction theory, (c) heat flow density of a material point in domain $\Omega^{+}$with other material points in domain $\Omega^{-}$, (d) heat flux density from domain $\Omega^{+}$due to domain $\Omega^{-}$

Fig. 3 Boundary layers for imposing temperature, heat flux, convection, and radiation Fig. 4 (a) Material point and its image in a fictitious domain (b) constant temperature condition (c) zero temperature condition

Fig. 5 Material point and its image in a fictitious region for imposing zero flux

Fig. 6 Discretization of one-dimensional region with collocation points

Fig. 7 Thermal interaction of points with the horizon of $i$

Fig. 8 Discretization of the finite slab and the fictitious boundary regions for temperatures

Fig. 9 Temperature variations from peridynamics and classical analytical solutions

Fig. 10 Discretization of the finite slab and boundary regions for convection

Fig. 11 Temperature variations from peridynamics and classical analytical solutions

Fig. 12 Peridynamic model of the plate

Fig. 13 Temperature variation from peridynamics and BEM at $y=0$ [24]

Fig. 14 Peridynamic model of a three-dimensional block

Fig. 15 Temperature variations from peridynamics and classical analytical solutions

Fig. 16 Peridynamic model of a plate with an insulated interface crack

Fig. 17 Temperature variations along $x=0$, across the interface of the plates with thermal conductivity $k_{1}$ for the upper half and $k_{2}$ for the lower half at $t=0.5 \mathrm{~s}$

Fig. 18 Three-dimensional peridynamic model of a plate with a crack

Fig. 19 Temperature field from two- and three-dimensional peridynamic analyses for $k_{1}=k_{2}=k$ at $t=0.5 \mathrm{~s}$ (two-dimensional model $=$ solid line, three-dimensional model $=$ dashed line)

Fig. 20 Peridynamic model of the thick plate: (a) boundary conditions type I; (b) boundary conditions type-II

Fig. 21 Three-dimensional peridynamic temperature predictions on the mid-plane with a normal in the $+z$ direction at $t=0.45 \mathrm{~s}$ for boundary conditions type-I $\left(\Theta_{0}=100^{\circ} \mathrm{C}\right)$

Fig. 22 Three-dimensional peridynamic temperature predictions on the mid-plane with a normal in the $+z$ direction at $t=0.45 \mathrm{~s}$ for boundary conditions type-II $\left(\Theta_{0}=100^{\circ} \mathrm{C}\right)$

Fig. A.1. Components of the position vector, $\xi$, between material points at $\mathbf{x}$ and $\mathbf{x}^{\prime}$.

Fig. C.1 Surface effects in the domain of interest

Fig. C.2 Material point $\mathbf{x}_{(i)}$ close to an interface 